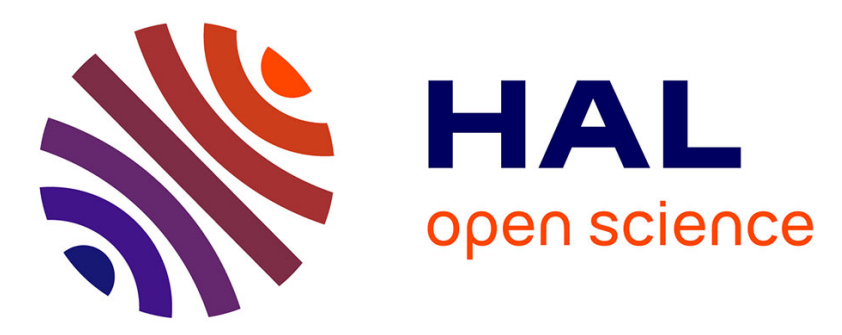

\title{
A sign matrix based scheme for non-homogeneous PDE's with an analysis of the convergence stagnation phenomenon
}

\author{
Slah Sahmim, Fayssal Benkhaldoun, Francisco Alcrudo
}

\section{To cite this version:}

Slah Sahmim, Fayssal Benkhaldoun, Francisco Alcrudo. A sign matrix based scheme for nonhomogeneous PDE's with an analysis of the convergence stagnation phenomenon. Journal of Computational Physics, 2007, 226 (2), pp.1753-1783. 10.1016/j.jcp.2007.06.017 . hal-00114522

\author{
HAL Id: hal-00114522 \\ https://hal.science/hal-00114522
}

Submitted on 17 Nov 2006

HAL is a multi-disciplinary open access archive for the deposit and dissemination of scientific research documents, whether they are published or not. The documents may come from teaching and research institutions in France or abroad, or from public or private research centers.
L'archive ouverte pluridisciplinaire $\mathbf{H A L}$, est destinée au dépôt et à la diffusion de documents scientifiques de niveau recherche, publiés ou non, émanant des établissements d'enseignement et de recherche français ou étrangers, des laboratoires publics ou privés. 


\title{
A sign matrix based scheme for non-homogeneous PDE's with an analysis of the convergence stagnation phenomenon
}

\author{
Slah Sahmim, Fayssal Benkhaldoun and Francisco Alcrudo \\ LAGA, Université Paris 13, 99 Av J.B. Clement, 93430 Villetaneuse, FRANCE \\ Email: fayssal,sahmim@math.univ-paris13.fr \\ CPS-Universidad de Zaragoza, Area de Mecanica de Fluidos, Maria de Luna, 3, \\ 50.015 Zaragoza, SPAIN \\ E-mail:alcrudo@unizar.es
}

\begin{abstract}
This work is devoted to the analysis of a finite volume method recently proposed for the numerical computation of a class of non homogenous systems of partial differencial equations of interest in fluid dynamics. The stability analysis of the proposed scheme leads to the introduction of the sign matrix of the flux jacobian. It appears that this formulation is equivalent to the VFRoe scheme introduced in the homogeneous case and has a natural extension here to non homogeneous systems. Comparative numerical experiments for the Shallow Water and Euler equations with source terms, and a model problem of two phase flow (Ransom faucet) are presented to validate the scheme. The numerical results present a convergence stagnation phenomenon for certain forms of the source term, notably when it is singular. Convergence stagnation has been also shown in the past for other numerical schemes. This issue is addressed in a specific section where an explanation is given with the help of a linear model equation, and a cure is demonstrated.
\end{abstract}

Key words: Finite volumes, Riemann Problems, SRNH scheme, Source terms, Shallow Water equations, Euler equations, Two-phase flow PACS: 03483, 38675, 41076

\section{Introduction}

Many interesting phenomena in the field of fluid dynamics are governed by systems of partial differential equations of the hyperbolic type. When they state a conservation law the corresponding system is homogeneous. If the 
system of equations expresses the rate at which some quantities are created or destroyed then it includes source terms. The general form of such a system in one spatial dimension can be written as:

$$
\frac{\partial W}{\partial t}+\frac{\partial F(W)}{\partial x}=Q(x, t, W)
$$

where $x$ is the space variable, $t$ the time, $W(x, t)$ is the variables vector, $F(W)$ the flux vector and $Q(x, t, W)$ the source vector. The sytem is to be solved subject to appropriate initial and boundary conditions:

$$
\begin{gathered}
W(x, 0)=u_{0}(x) \\
W(0, t)=u_{a}(t), \quad W(L, t)=u_{b}(t)
\end{gathered}
$$

The Shallow Water equations (SWE) describing one dimensional (1D) water flow in a channel with a variable bed and the Euler equations (EUL) describing 1D gas flow in a nozzle of variable cross section can be expressed as system (1). Note that when the channel bed (SWE) or the nozzle cross section (EUL) becomes discontinuous, the source term $Q$ becomes stiff, and system (1) becomes singular, hence the difficulty to numerically solve it. Also the common pressure multifluid model can be described by system (1). However it is well known that the flux jacobian of the common pressure multifluid model has characteristic roots that can become complex. This implies that the system of equations is no longer hyperbolic and the corresponding initial value problem may be ill posed.

From this remarks, one deduces the necessity of devising robust and efficient schemes to accurately solve non homogenous systems.

To this purpose, considerable work has been developed, during the last decade, to solve problems of the form (1-3) by finite volume methods.

Although upwind schemes were initially developed for the Euler equations, the number of papers devoted to the numerical solution of Shallow Water equations by means of Riemann solvers has greatly increased since the early works ([27], [2]). Specifically, efficient discretisations of source terms have been proposed by a large number of authors ([53], [45], [27])). In particular, one must mention the class of well-balanced schemes, designed to respect steady states. These schemes have been extensively studied by Le Roux and co-workers [28], [31], [32] in the scalar case, and in the framework of the SWE with topography in [11], [12], [13],[44] and with friction in [16]. Zhou and al. [63] proposed the surface gradient method for Shallow Water equations with source terms. Ming Tseng [47] presents different approches using TVD-MacCormack schemes, for the simulation of one dimensional open channel flows, with rapidly varying bottom topography. More recently, in [6], a well-balanced scheme is built based on local hydrostatic reconstructions. 
In [57], the authors propose a high order weighted essentially non-oscillatory finite volume scheme (WENO), and Runge-Kutta discontinuous Galerkin finite element methods (RKDG), for solving hyperbolic systems of conservation laws with source term.

Xing and Shu in [61] develop a well balanced high order finite volume WENO scheme for the SWE, wich is non-oscillatory, well balanced (satisfying the exact property) for still water, and genuinely high order in smooth regions. Other first and second order schemes have been applied to the SWE using the idea of balancing the source term and the flux gradients, for example the wave propagation algorithm by LeVeque [46], the kinetic scheme by $\mathrm{Xu}$ [62], and Perthame and Simeoni [48], central-upwind schemes proposed by Kurganov and Levy [39], and a family of flux-splitting numerical solvers proposed in [51]. It is worth pointing out that the contributions mentioned above, devoted to the numerical solution of hyperbolic problems with stiff source terms, do not address in general the question of convergence of the method, in particular when the system is singular.

In a different field, two phase flow problems raise numerous difficulties as regards the application of classical numerical methods. This is mainly due to non-linear interphase interactions. Finite Elements methods have been used in the context of two phase flows, when one is mainly interested in steady states, and wave effects are neglected [29].

Finite volume methods have been also extended to two phase problems. One way is to consider the mixture equilibrium model which enables the application of classical upwind schemes [30].

But in mixtures where the kinematic or thermal nonequilibrium is significant, one has to consider a system of equations for each phase, and two-fluid models have to be attacked [37]. In this case, the presence of non-conservative products introduces some conceptuel difficulties [19]. Nevertheless considerable improvements in the application of finite volumes to two phase flows have been achieved ([50], [56], [25], [15] [41]). For example in [59], the authors consider the case of a small difference between the liquid and gas velocities, and in [18], the case of a small ratio of gas to liquid densities, and in both cases these hypothesis are used to introduce some interesting simplifications. But only few works have been presented in the case of multidimensional twofluid problems in high non-equilibrium configurations ([26], [1]). These works present highly elaborate and efficient methods, dedicated specifically to two phase flows.

The challenge remains to devise finite volume schemes for general non homogeneous systems (either single or multiphase flows), which are at the same time accurate and stable, and not too CPU expensive.

The above mentioned appropriate finite volume schemes for two-phase flows, often rely upon approximate Riemann solvers that are computationally expensive due to the need for the calculation of exact or approximate jacobian field decompositions. 
In order to construct simpler and less expensive methods a particular class of non conservative problems is considered in this work: It is assumed that the solution to the Riemann problem associated with system (1) is self similar. This happens, for instance, if the source term can be written as:

$$
Q(x, W)=H(W) \frac{\partial G(x, W)}{\partial x} .
$$

where $H$ and $G$ are vector functions, which is the case for many problems of interest in fluid dynamics and in particular for the examples mentioned above. This work is a first step towards a multidimensional finite volume solver for non homogeneous systems, keeping in mind the need for simplicity, low cost and efficiency.

The non-homogeneous Riemann solver proposed in this paper (hereafter called $S R N H$ ) belongs to a large family of methods using only physical flux computations and average states instead of jacobian eigenvectors [58] and reveals to be both robust and accurate as well as computationally cheap. Moreover the extension to $2 \mathrm{D}$ calculations is easy and efficient ([9], [10]).

The $S R N H$ scheme depends upon a local diffusion controlling parameter. A mathematical analysis of the scheme both in the scalar equation, and in the case of a linear system of equations, shows that if one seeks a monotone scheme, this parameter can only be equal to a fixed value, which leads to the introduction of a sign matrix in the scheme. The method so constructed will be called $S R N H S$ in reference to the use of the sign matrix.

After giving a description of the method, its analysis is presented. As an illustration of the scheme efficiency, we present some results for singular problems like a dam break over a discontinuous bed, the shock tube problem of gas dynamics in a duct of discontinuous cross section and the classical Ransom Faucet problem in one space dimension. Results obtained show that the proposed scheme respects the well known equilibrium C-property for Shallowwater equations, and gives accurate solutions for the two-phase problem with few mesh points. It will also be shown that, in general, schemes not based upon exact Godunov solvers are not able to converge uniformly to the exact solution when the source function is singular although the numerical solution computed on a mesh of given finite size can be acceptable in many cases.

\section{Construction of $S R N H$ scheme}

Numerical approximations to the solution of (1-3) are sought within a finite volume formulation. For this end the domain of integration is discretised into finite volume cells. The approximate solution is assumed constant within each cell, i.e.: $u_{i}^{n}$ denotes the average of $W(x, t)$ within cell $i$ at time $t_{n}$. The length 
of cell $i$ is $\Delta x_{i}=x_{i+1 / 2}-x_{i-1 / 2}$ and the time step $\Delta t=t_{n+1}-t_{n}$. Unless otherwise specified, the mesh spacing will be assumed constant.

In deriving the scheme it will be assumed that the system admits an exact or approximate jacobian, which is strictly hyperbolic (diagonalisable in $\mathbb{R}$ ), and which is invertible. Nevertheless it will be shown that the method can also be applied to systems that are not strictly hyperbolic or that are hyperbolic only in a limited region of the phase space.

Let us recall the recent theoretical result of J.M. Hong [36]. Suppose $A(W)=$ $\frac{\partial F(W)}{\partial W}$ is diagonalizable in $\mathbb{R}$, with distinct eigenvalues $\lambda_{k}(W)$, such that $\lambda_{k}(W) \neq 0 \forall W, \forall k$; in case $G=G(x)$ is a Lipschitz continuous function of $x$, the Cauchy problem (1)-(2) admits a weak solution.

To establish the proof, the author uses Glimm's random choice method, constructs solutions of local Riemann problems of extended systems, and show convergence by regularisation.

The author shows that, provided the system is strictly hyperbolic, and for initial Riemann data close enough, the solution of a local Riemann problem, exists, is unique, self-similar, and consists of connecting left and right states by shock waves, contact discontinuities, rarefaction waves and a stationary wave discontinuity.

In a Godunov type scheme the solution is advanced in time by solving the Riemann problems that arise at cell interfaces after discretisation. Assume the self similar solution to the Riemann problem associated to equation (1) with initial conditions:

$$
W(x, 0)=u_{0}(x)=\left\{\begin{array}{l}
u_{L} \text { if } x<0 \\
u_{R} \text { if } x>0
\end{array}\right.
$$

is:

$$
W(x, t)=R_{s}\left(\frac{x}{t}, u_{L}, u_{R}, G\right)
$$

For some forms of the function $G(x, W)$ the Riemann solution $R_{s}$ can be exactly computed (see [3] for the Shallow Water equations, [43] for the isentropic Euler equations and [5] for the full non-isentropic Euler equations).

Integration of equation (1) over the domain $\left[x_{i-1 / 2}, x_{i+1 / 2}\right] \times\left[t_{n}, t_{n+1}\right]$ leads to:

$$
W_{i}^{n+1}=W_{i}^{n}-\frac{\Delta t}{\Delta x}\left[F\left(W_{i+\frac{1}{2}}^{n}\right)-F\left(W_{i-\frac{1}{2}}^{n}\right)\right]+\Delta t Q_{i}^{n}
$$

where $W_{i+1 / 2}$ is, in principle, the Riemann solution at cell interface $i+1 / 2$ :

$$
W_{i+1 / 2}=R_{s}\left(0, W_{i}, W_{i+1}, G\left(x-x_{i+1 / 2}\right)\right)
$$




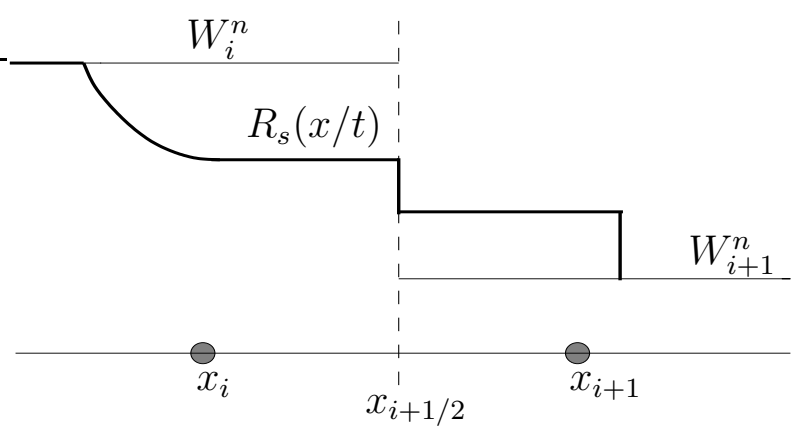

Fig. 1. The Riemann problem solution at a cell interface.

and $Q_{i}^{n}$ an approximation of:

$$
Q_{i}^{n}=\frac{1}{\Delta x \Delta t} \int_{t_{n}}^{t_{n+1}} \int_{x_{i-\frac{1}{2}}}^{x_{i+\frac{1}{2}}} Q(x, W) d x d t
$$

The practical calculation of $R_{s}$ can be computationally very expensive for arbitrary initial data. Moreover, discretisation of the source term can lead to singularities at the cell interface for some forms of $G(x, W)$. This makes $R_{s}$ become discontinuous at the interface (see figure 1 ).

In order to avoid these difficulties and find an estimate for $u_{i+1 / 2}$ at less cost, the system of differential equations is integrated again, this time over the domain $\left[X^{-}, X^{+}\right] \times\left[t_{n}, t_{n}+\theta_{i+1 / 2}^{n}\right]$ that contains $x_{i+1 / 2}$ as figure 2 shows. In general the following we will take: $X^{-}=x_{i}$ and $X^{+}=x_{i+1}$, but other choices could also be considered. This leads to:

$$
W_{i+1 / 2}^{n}=\frac{1}{2}\left(W_{i}^{n}+W_{i+1}^{n}\right)-\frac{\theta_{i+1 / 2}^{n}}{\Delta x}\left[F\left(W_{i+1}^{n}\right)-F\left(W_{i}^{n}\right)\right]+\theta_{i+1 / 2}^{n} Q_{i+1 / 2}^{n}
$$

where $u_{i+1 / 2}^{n}$ can be thought of now as an approximation to the average of the Riemann solution $R_{s}$ over the length $\left[x_{i}, x_{i+1}\right]$ at time $\theta_{i+1 / 2}^{n}$. By doing so any discontinuities in $W$ at the cell interface are smeared out.

Also $Q_{i+1 / 2}^{n}$ must be evaluated as an approximation to:

$$
\frac{1}{\Delta x \theta_{n+1 / 2}} \int_{x_{i}}^{x_{i+1}} \int_{t_{n}}^{t_{n}+\theta_{n+1 / 2}} Q(x, W) d x d t
$$

in a judicious way (to be dealt with later).

In [8], $\theta_{i+1 / 2}^{n}$ is taken as a fraction of the current time step:

$$
\theta_{i+1 / 2}^{n}=\alpha_{i+1 / 2}^{n} \frac{\Delta t}{2}
$$




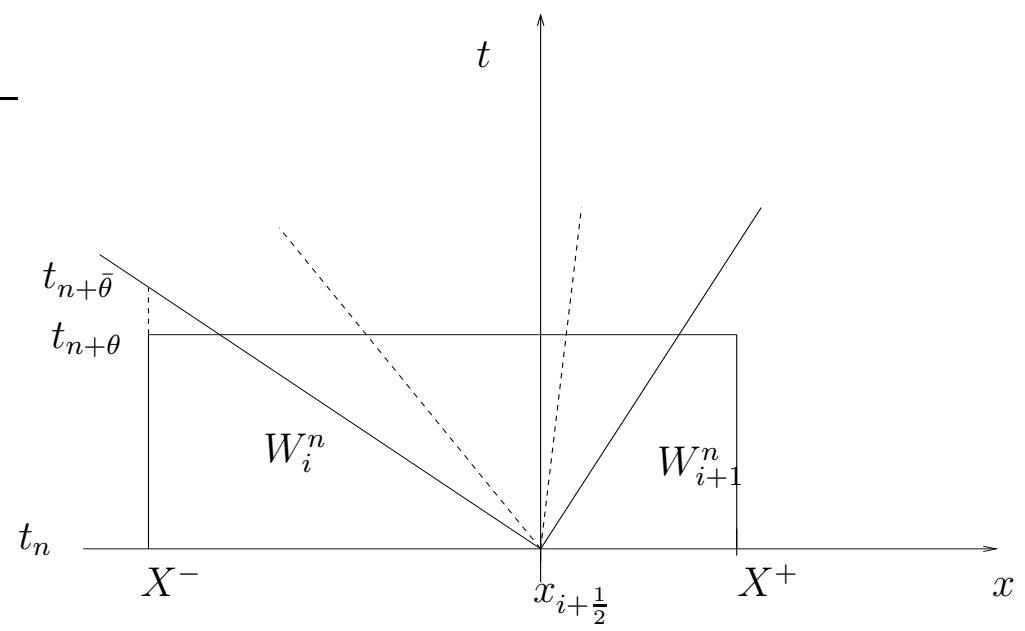

Fig. 2. Integration of the equations around cell-edge $x_{i+1 / 2}$

Where $\alpha_{i+1 / 2}^{n}$ is a real positive number that remains arbitrary in principle.

It is worth noting that in view of (12), the evaluation of $W_{i+1 / 2}^{n}$ (the predictor or intermediate state) is made with a local time step. Also, at least in principle, in order that equation (10) be consistent, $\theta_{i+1 / 2}^{n}$ should not be larger than a certain limit $\bar{\theta}_{i+1 / 2}^{n}$ that corresponds to the time taken by the fastest wave generated at $x_{i+1 / 2}$ to leave the cell $\left[X^{-}=x_{i}, X^{+}=x_{i+1}\right]$ (see figure 2 ):

$$
\theta_{i+1 / 2}^{n} \leq \bar{\theta}_{i+1 / 2}^{n}, \quad \bar{\theta}_{i+1 / 2}^{n}=\frac{\Delta x}{2 S_{i+1 / 2}^{n}}
$$

where $S_{i+1 / 2}^{n}$ is an approximation to the modulus of the velocity of the fastest wave in the system. Otherwise the flux at either $x_{i}$ or $x_{i+1}$ could not be taken as $F\left(u_{i}\right)$ or $F\left(u_{i+1}\right)$ respectively. Since it is costly to obtain the exact solution of the Riemann problem at $x_{i+1 / 2}$ and hence the speed of the waves, an approximation to $S_{i+1 / 2}^{n}$ can be taken as the local Rusanov velocity [54]:

$$
S_{i+\frac{1}{2}}^{n}=\max _{p=1, \ldots, m}\left(\max \left(\left|\lambda_{p, i}^{n}\right|,\left|\lambda_{p, i+1}^{n}\right|\right)\right)
$$

$\lambda_{p, i}^{n}$ being the $p^{t h}$ eigenvalue of the flux jacobian evaluated at the state $W_{i}^{n}$. In view of this definition one can simply take $\theta_{i+1 / 2}^{n}$ as a fraction of $\bar{\theta}_{i+1 / 2}^{n}$ :

$$
\theta_{i+1 / 2}^{n}=\alpha_{i+1 / 2}^{n} \bar{\theta}_{i+1 / 2}^{n}
$$

what gives simply:

$$
\theta_{i+1 / 2}^{n}=\frac{\Delta x}{2} \frac{\alpha_{i+1 / 2}^{n}}{S_{i+1 / 2}^{n}}
$$

The analysis performed in next section will provide the appropriate expression for $\alpha_{i+1 / 2}^{n}$ that makes the global scheme fulfill certain stability properties. The choice (16) instead of (12) has considerable advantages when the scheme is extended to two dimensions since it makes cancel the $\Delta x$ factor in both the 
flux difference and the source term. Recall that in this work we are dealing with source terms of the form (4) that, after discretisation, give rise to expressions of the form:

$$
Q_{i+1 / 2}^{n}=H_{i+1 / 2}^{n} \frac{G_{i+1}^{n}-G_{i}^{n}}{\Delta x}
$$

Hence the $S R N H$ scheme can be formally written:

$$
\left\{\begin{aligned}
W_{i+\frac{1}{2}}^{n} & =\frac{1}{2}\left(W_{i+1}^{n}+W_{i}^{n}\right)-\frac{\alpha_{i+\frac{1}{2}}^{n}}{2 S_{i+\frac{1}{2}}^{n}}\left(F\left(W_{i+1}^{n}\right)-F\left(W_{i}^{n}\right)\right) \\
& +\frac{\alpha_{i+\frac{1}{2}}^{n}}{2 S_{i+\frac{1}{2}}^{n}} H_{i+1 / 2}^{n}\left(G_{i+1}^{n}-G_{i}^{n}\right) \\
W_{i}^{n+1} & =W_{i}^{n}-\frac{\Delta t}{\Delta x}\left(F\left(W_{i+\frac{1}{2}}^{n}\right)-F\left(W_{i-\frac{1}{2}}^{n}\right)\right) \\
& +\frac{\Delta t}{2 \Delta x} H_{i}^{n}\left(G_{i+1}^{n}-G_{i-1}^{n}\right)
\end{aligned}\right.
$$

$H_{i+1 / 2}^{n}$ and $H_{i}^{n}$ being judicious approximations of $H$ at interface $x_{i+1 / 2}$ and cell $i$ respectively, and $\alpha_{i+1 / 2}^{n}$ remains a local parameter that must be chosen.

Remark

that the $S R N H$ scheme appears as an upwind method in which instead of upwinding the numerical flux, the variables are upwinded to construct an average state at the interface, $W_{i+1 / 2}^{n}$, which is then used in the physical flux and source functions. The hyperbolic character of the system is not strongly needed here.

\section{Analysis of $S R N H$ scheme in the scalar case}

The choice of $\alpha_{i+1 / 2}^{n}$ in the $S R N H$ scheme remains arbitrary. For instance in the linear case if $\alpha=1$, the $S R N H$ scheme reduces to first order upwind method and for $\alpha=a \Delta t / \Delta x$, it falls back to Lax-Wendroff method. For large values of alpha, the numerical diffusion of the scheme correspondingly increases. A careful choice of the parameter can furnish the scheme (in the homogeneous case) with desirable properties such as stability, monotonicity and a maximum principle. In the following an anlysis of $S R N H$ scheme is made in the homogeneous case, with the aim of ensuring the above mentioned properties. 
In the case of a homogeneous scalar equation, $S R N H$ scheme can be written:

$$
\left\{\begin{array}{l}
u_{i+\frac{1}{2}}^{n}=\frac{1}{2}\left(u_{i+1}^{n}+u_{i}^{n}\right)-\frac{\alpha_{i+\frac{1}{2}}^{n}}{2 S_{i+\frac{1}{2}}^{n}}\left(f\left(u_{i+1}^{n}\right)-f\left(u_{i}^{n}\right)\right) \\
u_{i}^{n+1}=u_{i}^{n}-r\left(f\left(u_{i+\frac{1}{2}}^{n}\right)-f\left(u_{i-\frac{1}{2}}^{n}\right)\right)
\end{array}\right.
$$

where $S_{i+\frac{1}{2}}^{n}=\max \left(\left|f^{\prime}\left(u_{i+1}^{n}\right)\right|,\left|f^{\prime}\left(u_{i}^{n}\right)\right|\right)$ is the local Rusanov velocity, and $r=$ $\Delta t / \Delta x$.

Proposition 1 Suppose $f$ is a monotone $C^{1}$ function, then under the two conditions:

$$
\begin{aligned}
& \text { i) } \frac{S_{i+1 / 2}^{n}}{\left|f^{\prime}\left(a_{i+1 / 2}^{n}\right)\right|} \leq \alpha_{i+1 / 2}^{n} \leq \gamma \frac{S_{i+1 / 2}^{n}}{\left|f^{\prime}\left(a_{i+1 / 2}^{n}\right)\right|}, \quad \forall i \in \mathbb{Z}, n \in \mathbb{N} \\
& \text { ii) } \quad r \gamma A \leq 1
\end{aligned}
$$

where: $\gamma \geq 1, A=\max \left|f^{\prime}(X)\right|$ such that $|X| \leq \gamma|| u_{0} \|_{L^{\infty}(\mathbb{R})}$, and $a_{i+1 / 2}^{n}$ is a Roe state, determined by the mean value theorem:

$$
f\left(u_{i+1}^{n}\right)-f\left(u_{i}^{n}\right)=f^{\prime}\left(a_{i+1 / 2}^{n}\right)\left(u_{i+1}^{n}-u_{i}^{n}\right),
$$

the scheme (19) respects the local maximum principle:

$$
\min _{i \in \mathbb{Z}} u_{i}^{n} \leq \min _{i \in \mathbb{Z}} u_{i}^{n+1} \leq \max _{i \in \mathbb{Z}} u_{i}^{n+1} \leq \max _{i \in \mathbb{Z}} u_{i}^{n} .
$$

PROOF. The result is obtained by writing

$$
\begin{gathered}
\delta_{i+\frac{1}{2}}^{n}=\frac{\alpha_{i+\frac{1}{2}}^{n}}{s_{i+\frac{1}{2}}^{n}} f^{\prime}\left(a_{i+\frac{1}{2}}^{n}\right) \\
u_{i+\frac{1}{2}}^{n}=\frac{1}{2}\left[1+\delta_{i+\frac{1}{2}}^{n}\right] u_{i}^{n}+\frac{1}{2}\left[1-\delta_{i+\frac{1}{2}}^{n}\right] u_{i+1}^{n}
\end{gathered}
$$

and

$$
u_{i}^{n+1}=u_{i}^{n}-r f^{\prime}\left(a_{i}^{n}\right)\left(u_{i+\frac{1}{2}}^{n}-u_{i-\frac{1}{2}}^{n}\right)
$$

Then expressing $u_{i}^{n+1}$ as a convex combination of $u_{i-1}^{n}, u_{i}^{n}$, and $u_{i+1}^{n}$ ends the proof.

Proposition 2 Suppose that $f^{\prime}$ does not change sign. Under the two following conditions:

1) $\quad \alpha_{i+1 / 2}^{n}=\bar{\gamma} \frac{S_{i+1 / 2}^{n}}{\left|f^{\prime}\left(a_{i+1 / 2}^{n}\right)\right|}, \forall i \in \mathbb{Z}, n \in \mathbb{N}$ with $\bar{\gamma}$ a constant, and $\bar{\gamma} \in[1, \gamma]$, 
2) $\quad r \gamma A \leq 1$.

Then scheme (19) is monotone.

PROOF. A sufficient condition for fulfilling the first condition is to fix $\bar{\gamma} \in$ $[1, \gamma]$ and choose:

$$
\alpha_{i+1 / 2}^{n}=\bar{\gamma} \frac{S_{i+1 / 2}^{n}}{\left|f^{\prime}\left(a_{i+1 / 2}^{n}\right)\right|} .
$$

In such case the predictor step becomes:

$$
u_{i+\frac{1}{2}}^{n}=\frac{1}{2}\left(1+\bar{\gamma} \operatorname{sgn}\left(f^{\prime}\right)\right) u_{i}^{n}+\frac{1}{2}\left(1-\bar{\gamma} \operatorname{sgn}\left(f^{\prime}\right)\right) u_{i+1}^{n}
$$

and the corrector step can be written in the following conservative form:

$$
\begin{aligned}
u_{i}^{n+1} & =u_{i}^{n}-r\left(f\left(u_{i+1 / 2}^{n}\right)-f\left(u_{i-1 / 2}^{n}\right)\right), \\
& =\mathcal{H}\left(u_{i-1}^{n}, u_{i}^{n}, u_{i+1}^{n}\right)
\end{aligned}
$$

The scheme (27) is monotone if operator $\mathcal{H}$ is increasing with respect to each one of its arguments: $u_{i}^{n}, u_{i-1}^{n}$ and $u_{i+1}^{n}[21]$.

$$
\begin{gathered}
\frac{\partial \mathcal{H}}{\partial u_{i-1}^{n}}\left(u_{i-1}^{n}, u_{i}^{n}, u_{i+1}^{n}\right)=\frac{r}{2}\left(\operatorname{sgn}\left(f^{\prime}\right)+\bar{\gamma}\right)\left|f^{\prime}\left(a_{i}^{n}\right)\right|, \\
\frac{\partial \mathcal{H}}{\partial u_{i+1}^{n}}\left(u_{i-1}^{n}, u_{i}^{n}, u_{i+1}^{n}\right)=-\frac{r}{2}\left(\operatorname{sgn}\left(f^{\prime}\right)-\bar{\gamma}\right)\left|f^{\prime}\left(a_{i}^{n}\right)\right|, \\
\frac{\partial \mathcal{H}}{\partial u_{i}^{n}}\left(u_{i-1}^{n}, u_{i}^{n}, u_{i+1}^{n}\right)=1-r \bar{\gamma} f^{\prime}\left(a_{i}^{n}\right) .
\end{gathered}
$$

Since $\bar{\gamma} \geq 1$ and $r \bar{\gamma} A \leq 1$, the operator $\mathcal{H}$ is an increasing function and thus it follows that the scheme $S R N H$ is monotone.

Remark 3 The truncation error of the scheme in the homogeneous case is:

$$
T E(u)(x, t)=-\Delta t \frac{\partial}{\partial x}\left[B \frac{\partial u}{\partial x}\right]
$$


where:

$$
B=\frac{\left|f^{\prime}(u)\right|}{2 r}\left(\bar{\alpha}(u)-r\left|f^{\prime}(u)\right|\right)
$$

where one assumes $\alpha_{i+1 / 2}^{n}=\alpha\left(u_{i}^{n}, u_{i+1}^{n}\right)$, and $\lim _{u_{1}, u_{2} \rightarrow u}\left(\alpha\left(u_{1}, u_{2}\right)\right)=\bar{\alpha}(u)$.

That clearly shows $\alpha_{i+1 / 2}^{n}$ as a parameter controlling the local numerical diffusion, and if $\alpha_{i+1 / 2}^{n}$ is close to the CFL number, then the SRNH scheme is quasi-second order accurate.

\subsection{Optimization of parameter $\alpha$}

Remark that the second part of the first condition of Proposition (2) above, is to ensure that the state $u_{i+\frac{1}{2}}^{n}$ and hence $a_{i}^{n}$ remains finite. Instead of this one can impose the sufficient but more restrictive condition that the intermediate state $u_{i+1 / 2}^{n}$ remains between the minimum and maximum of states $u_{i}^{n}$ and $u_{i+1}^{n}$. From (22) this is realized if: $1+\delta_{i+\frac{1}{2}}^{n} \geq 0$ and $1-\delta_{i+\frac{1}{2}}^{n} \geq 0$. Then it follows that:

$$
\left|\delta_{i+\frac{1}{2}}^{n}\right| \leq 1 \quad \Longleftrightarrow \quad \alpha_{i+\frac{1}{2}}^{n} \leq \frac{S_{i+\frac{1}{2}}^{n}}{\left|f^{\prime}\left(a_{i+\frac{1}{2}}^{n}\right)\right|} .
$$

which is equivalent to putting $\gamma=1$ in $i$ ) of Proposition (1) and then:

$$
\alpha_{i+\frac{1}{2}}^{n}=\frac{S_{i+\frac{1}{2}}^{n}}{\left|f^{\prime}\left(a_{i+\frac{1}{2}}^{n}\right)\right|}
$$

Substituting back in (19) and using (20) gives the following form for the $S R N H$ scheme:

$$
\left\{\begin{array}{l}
u_{i+\frac{1}{2}}^{n}=\frac{1}{2}\left(u_{i+1}^{n}+u_{i}^{n}\right)-\frac{1}{2} \operatorname{sgn}\left[f^{\prime}\left(a_{i+\frac{1}{2}}^{n}\right)\right]\left(u_{i+1}^{n}-u_{i}^{n}\right) \\
u_{i}^{n+1}=u_{i}^{n}-r\left(f\left(u_{i+\frac{1}{2}}^{n}\right)-f\left(u_{i-\frac{1}{2}}^{n}\right)\right)
\end{array}\right.
$$

where $\operatorname{sgn}(x)$ stands for:

$$
\operatorname{sgn}(\mathrm{x})=\left\{\begin{array}{ccc}
\frac{x}{|x|} & \text { if } \quad x \neq 0 \\
0 & \text { if } \quad x=0
\end{array}\right.
$$

It appears that the predictor step leads to the upwinding of the variables in a similar way to the VFRoe scheme [22]. 


\section{Linear homogeneous systems}

The following linear strictly hyperbolic system is considered:

$$
\left\{\begin{array}{l}
\frac{\partial W}{\partial t}+\mathcal{A} \frac{\partial W}{\partial x}=0,(x, t) \in \mathcal{D} \times \mathbb{R}_{+}^{*}, \mathcal{D} \subset \mathbb{R} \\
W(x, 0)=u_{0}(x), x \in \mathcal{D}
\end{array}\right.
$$

with $W: \mathcal{D} \times \mathbb{R}_{+} \rightarrow \Omega$ a vector of $m$ conserved quantities, or state variables, $\Omega$ is a bounded open domain in $\mathbb{R}^{m}, \mathcal{A}$ is a square matrix $\mathcal{M}_{m}(\mathbb{R})$.

We denote $\lambda_{1} \leq \lambda_{2} \leq \ldots \leq \lambda_{m}$ the eigenvalues associated to the eigenvectors $\left\{r_{1}, \ldots, r_{m}\right\}$, and denote $\overline{\mathcal{B}}=\left\{r_{1}, \ldots, r_{m}\right\}$ the base of $\mathbb{R}^{m}$ eigenvectors of $\mathcal{A}$. Taking $\mathcal{R}=\left[r_{1}, \ldots, r_{m}\right]$, it follows that $\mathcal{A}=\mathcal{R} \Lambda \mathcal{R}^{-1}$, where $\Lambda$ is the diagonal form of $\mathcal{A}$ and $\mathcal{R}^{-1}$ is the inverse of $\mathcal{R}$.

For this problem the $S R N H$ scheme presented in [8] and [55] reads:

$$
\left\{\begin{array}{l}
W_{i+\frac{1}{2}}^{n}=\frac{1}{2}\left(W_{i+1}^{n}+W_{i}^{n}\right)-\frac{\alpha_{i+\frac{1}{2}}^{n}}{2 S_{i+\frac{1}{2}}^{n}} \mathcal{A}\left(W_{i+1}^{n}-W_{i}^{n}\right) \\
W_{i}^{n+1}=W_{i}^{n}-r \mathcal{A}\left(W_{i+\frac{1}{2}}^{n}-W_{i-\frac{1}{2}}^{n}\right)
\end{array}\right.
$$

where

$$
S_{i+\frac{1}{2}}^{n}=\max _{p=1, \ldots, m}\left(\left|\lambda_{p}\right|\right)=\rho(\mathcal{A}),
$$

with $\rho(\mathcal{A})$ the spectral radius of $\mathcal{A}$ and $\alpha_{i+\frac{1}{2}}^{n}$ is a control parameter as before.

Writing the above system in characteristic form $\left(V=R^{-1} W\right)$, one gets:

$$
\left\{\begin{array}{l}
V_{i+\frac{1}{2}}^{n}=\frac{1}{2}\left(V_{i+1}^{n}+V_{i}^{n}\right)-\frac{\alpha_{i+\frac{1}{2}}^{n}}{2 S_{i+\frac{1}{2}}^{n}} \Lambda\left(V_{i+1}^{n}-V_{i}^{n}\right), \\
V_{i}^{n+1}=V_{i}^{n}-r \Lambda\left(V_{i+\frac{1}{2}}^{n}-V_{i-\frac{1}{2}}^{n}\right)
\end{array}\right.
$$

which, for each component $v_{p}$, leads to:

$$
\left\{\begin{array}{l}
\left(v_{p}\right)_{i+\frac{1}{2}}^{n}=\frac{1}{2}\left(\left(v_{p}\right)_{i+1}^{n}+\left(v_{p}\right)_{i}^{n}\right)-\frac{\alpha_{i+\frac{1}{2}}^{n}}{2 S_{i+\frac{1}{2}}^{n}} \lambda_{p}\left(\left(v_{p}\right)_{i+1}^{n}-\left(v_{p}\right)_{i}^{n}\right), \\
\left(v_{p}\right)_{i}^{n+1}=\left(v_{p}\right)_{i}^{n}-r \lambda_{p}\left(\left(v_{p}\right)_{i+\frac{1}{2}}^{n}-\left(v_{p}\right)_{i-\frac{1}{2}}^{n}\right) .
\end{array}\right.
$$

for $p=1, \ldots, m$.

This is the scheme $S R N H$ applied to the characteristic unknowns $v_{p}$.

Proposition 4 Under the two following conditions: 
1) $\alpha_{i+\frac{1}{2}}^{n}=\operatorname{Diag}\left(\alpha_{i+\frac{1}{2}, p}^{n}\right)$ where $\alpha_{i+\frac{1}{2}, p}^{n}=\frac{S_{i+\frac{1}{2}}^{n}}{\left|\lambda_{p}\right|}, \forall(i, n) \in \mathbb{Z} \times \mathbb{N}, \forall p \in\{1, \ldots, m\}$ such that $\lambda_{p} \neq 0$,

2) $r \rho(\mathcal{A}) \leq 1$.

the scheme satisfies the two extremum principles.

$$
\begin{aligned}
& \min _{i \in \mathbb{Z}}\left(v_{p}\right)_{i}^{n} \leq \min _{i \in \mathbb{Z}}\left(v_{p}\right)_{i}^{n+1} \leq \max _{i \in \mathbb{Z}}\left(v_{p}\right)_{i}^{n+1} \leq \max _{i \in \mathbb{Z}}\left(v_{p}\right)_{i}^{n} . \\
& \min \left(\left(v_{p}\right)_{i}^{n},\left(v_{p}\right)_{i+1}^{n}\right) \leq\left(v_{p}\right)_{i+\frac{1}{2}}^{n} \leq \max \left(\left(v_{p}\right)_{i}^{n},\left(v_{p}\right)_{i+1}^{n}\right)
\end{aligned}
$$

\section{Proof}

The proof follows from using the analysis of section 3 and writing the $S R N H$ scheme for the characteristic unknowns $v_{p}$ under the form (36).

One then finds that the control parameter $\alpha_{i+\frac{1}{2}}^{n}$ depends on the local eigenvalue for each characteristic equation. Hence it is clear that for the overall system it must have a diagonal matrix form:

$$
\alpha_{i+\frac{1}{2}}^{n}=\left(\begin{array}{cccc}
\frac{S_{i+\frac{1}{2}}^{n}}{\left|\lambda_{1}\right|} & 0 & \ldots & 0 \\
0 & \frac{S_{i+\frac{1}{2}}^{n}}{\left|\lambda_{2}\right|} & \ldots & \vdots \\
\vdots & 0 & \ddots & 0 \\
& & & \\
0 & \ldots & 0 & \frac{S_{i+\frac{1}{2}}^{n}}{\left|\lambda_{m}\right|}
\end{array}\right)=S_{i+\frac{1}{2}}^{n}|\Lambda|^{-1}
$$

Going back to the conservative form $(W=\mathcal{R} V)$ one gets:

$$
\left\{\begin{array}{l}
W_{i+\frac{1}{2}}^{n}=\frac{1}{2}\left(W_{i+1}^{n}+W_{i}^{n}\right)-\frac{1}{2} \operatorname{sgn}(\mathcal{A})\left(W_{i+1}^{n}-W_{i}^{n}\right), \\
W_{i}^{n+1}=W_{i}^{n}-r\left(F\left(W_{i+\frac{1}{2}}^{n}\right)-F\left(W_{i-\frac{1}{2}}^{n}\right)\right)
\end{array}\right.
$$

with $\operatorname{sgn}(\mathcal{A})=\mathcal{R} \operatorname{sgn}(\Lambda) \mathcal{R}^{-1}$ and $\operatorname{sgn}(\Lambda)=\operatorname{diag}\left(\frac{\lambda_{k}}{\left|\lambda_{k}\right|}\right)$. 


\section{Extension of SRNHS scheme to non homogeneous non linear hyperbolic systems}

The $S R N H$ scheme (18) can be directly applied to system (1), but $\alpha_{i+1 / 2}^{n}$ must be optimized as it has been done in the linear case. In order to follow the strategy of previous section, the problem is first localy linearized. We suppose that there exists an average state $\bar{W}\left(W_{i}^{n}, W_{i+1}^{n}\right)$, such that the relation:

$$
F\left(W_{i+1}^{n}\right)-F\left(W_{i}^{n}\right)=A\left(\bar{W}\left(W_{i}^{n}, W_{i+1}^{n}\right)\right)\left(W_{i+1}^{n}-W_{i}^{n}\right) .
$$

is realized; for instance the Roe average state [52] of the system verifies exactly this property. The predictor step becomes then:

$$
\begin{aligned}
W_{i+\frac{1}{2}}^{n}=\frac{1}{2}\left(W_{i+1}^{n}+W_{i}^{n}\right)- & \frac{\alpha_{i+\frac{1}{2}}^{n}}{2 S_{i+\frac{1}{2}}^{n}} \bar{A}_{i+1 / 2}^{n}\left(W_{i+1}^{n}-W_{i}^{n}\right) \\
& +\frac{\alpha_{i+\frac{1}{2}}^{n}}{2 S_{i+\frac{1}{2}}^{n}} H_{i+1 / 2}^{n}\left(G_{i+1}^{n}-G_{i}^{n}\right)
\end{aligned}
$$

where

$$
\bar{A}_{i+1 / 2}^{n}=A\left(\bar{W}\left(W_{i}^{n}, W_{i+1}^{n}\right)\right)=\bar{R}_{i+\frac{1}{2}}^{n} \bar{\Lambda}_{i+\frac{1}{2}}^{n}\left(\bar{R}_{i+\frac{1}{2}}^{n}\right)^{-1}
$$

In analogy with the linear case we introduce the local characteristic variables $V=R^{-1} W$ around cell interface $x_{i+1 / 2}$ as follows:

$$
\begin{aligned}
V_{i+1 / 2}^{n} & =\left(\bar{R}_{i+\frac{1}{2}}^{n}\right)^{-1} W_{i+1 / 2}^{n} \\
V_{i+1 / 2}^{n,-} & =\left(\bar{R}_{i+\frac{1}{2}}^{n}\right)^{-1} W_{i}^{n} \\
V_{i+1 / 2}^{n,+} & =\left(\bar{R}_{i+\frac{1}{2}}^{n}\right)^{-1} W_{i+1}^{n}
\end{aligned}
$$

what leads to the following expression for the predictor step of $S R N H$ scheme for the local charateristic variables:

$$
\begin{array}{r}
V_{i+\frac{1}{2}}^{n}=\frac{1}{2}\left(V_{i+1 / 2}^{n,+}+V_{i+1 / 2}^{n,-}\right)-\frac{\alpha_{i+\frac{1}{2}}^{n}}{2 S_{i+\frac{1}{2}}^{n}} \bar{\Lambda}_{i+1 / 2}^{n}\left(V_{i+1 / 2}^{n,+}-V_{i+1 / 2}^{n,-}\right) \\
+\frac{\alpha_{i+\frac{1}{2}}^{n}}{2 S_{i+\frac{1}{2}}^{n}}\left(\bar{R}_{i+\frac{1}{2}}^{n}\right)^{-1} H_{i+1 / 2}^{n}\left(G_{i+1}^{n}-G_{i}^{n}\right)
\end{array}
$$

Following the same reasoning as in the linar system case, it turns out that $\alpha$ must be a matrix of the form $\alpha_{i+\frac{1}{2}}^{n}=S_{i+\frac{1}{2}}^{n}\left|\bar{\Lambda}_{i+\frac{1}{2}}\right|^{-1}$ what leads to:

$$
\begin{array}{r}
V_{i+\frac{1}{2}}^{n}=\frac{1}{2}\left(V_{i+1 / 2}^{n,+}+V_{i+1 / 2}^{n,-}\right)-\frac{1}{2}\left|\bar{\Lambda}_{i+\frac{1}{2}}\right|^{-1} \bar{\Lambda}_{i+1 / 2}^{n}\left(V_{i+1 / 2}^{n,+}-V_{i+1 / 2}^{n,-}\right) \\
+\frac{1}{2}\left|\bar{\Lambda}_{i+\frac{1}{2}}\right|^{-1}\left(\bar{R}_{i+\frac{1}{2}}^{n}\right)^{-1} H_{i+1 / 2}^{n}\left(G_{i+1}^{n}-G_{i}^{n}\right)
\end{array}
$$


then coming back to the conservative variables:

$$
\begin{aligned}
W_{i+\frac{1}{2}}^{n}=\frac{1}{2}\left(W_{i+1}^{n}+W_{i}^{n}\right) & -\frac{1}{2} \bar{R}_{i+\frac{1}{2}}^{n}\left|\bar{\Lambda}_{i+\frac{1}{2}}\right|^{-1} \bar{\Lambda}_{i+1 / 2}^{n}\left(\bar{R}_{i+\frac{1}{2}}^{n}\right)^{-1}\left(W_{i+1}^{n}-W_{i}^{n}\right) \\
& +\frac{1}{2} \bar{R}_{i+\frac{1}{2}}^{n}\left|\bar{\Lambda}_{i+\frac{1}{2}}\right|^{-1}\left(\bar{R}_{i+\frac{1}{2}}^{n}\right)^{-1} H_{i+1 / 2}^{n}\left(G_{i+1}^{n}-G_{i}^{n}\right)
\end{aligned}
$$

the SRNHS scheme for problem (1) can thus be written:

$$
\left\{\begin{aligned}
W_{i+\frac{1}{2}}^{n}=\frac{1}{2}\left(W_{i+1}^{n}+\right. & \left.W_{i}^{n}\right)-\frac{1}{2} \operatorname{sgn}\left[\bar{A}_{i+1 / 2}^{n}\right]\left(W_{i+1}^{n}-W_{i}^{n}\right) \\
& +\frac{1}{2}\left|\bar{A}_{i+1 / 2}^{n}\right|^{-1} H_{i+1 / 2}^{n}\left(G_{i+1}^{n}-G_{i}^{n}\right) \\
W_{i}^{n+1}=W_{i}^{n}-\frac{\Delta t}{\Delta x} & \left(F\left(W_{i+\frac{1}{2}}^{n}\right)-F\left(W_{i-\frac{1}{2}}^{n}\right)\right) \\
& +\frac{\Delta t}{2 \Delta x} H_{i}^{n}\left(G_{i+1}^{n}-G_{i-1}^{n}\right)
\end{aligned}\right.
$$

where

$$
\operatorname{sgn}\left[\bar{A}_{i+1 / 2}^{n}\right]=\bar{R}_{i+\frac{1}{2}}^{n}\left|\bar{\Lambda}_{i+\frac{1}{2}}^{n}\right|^{-1} \bar{\Lambda}_{i+\frac{1}{2}}^{n}\left(\bar{R}_{i+\frac{1}{2}}^{n}\right)^{-1}
$$

and

$$
\left|\bar{A}_{i+1 / 2}^{n}\right|^{-1}=\bar{R}_{i+\frac{1}{2}}^{n}\left|\bar{\Lambda}_{i+\frac{1}{2}}^{n}\right|^{-1}\left(\bar{R}_{i+\frac{1}{2}}^{n}\right)^{-1}
$$

The particular approximation used to discretize the source term, $H_{i+1 / 2}^{n}$ and $H_{i}^{n}$ above, are left open until the particular system of equations to be solved is chosen in order to fulfill particular steady state properties of the system considered.

\section{Remark}

The practice shows that the simple classical average state:

$$
\bar{W}\left(W_{i}^{n}, W_{i+1}^{n}\right)=\frac{1}{2}\left(W_{i+1}^{n}+W_{i}^{n}\right) .
$$

can be used instead of the exact Roe state at considerably less computational cost.

\section{Application of the $S R N H S$ scheme to the Shallow Water Equa- tions with irregular topography}

We consider here the movement of a layer of fluid in one dimension over a fixed bed. The bed elevation with respect to a fixed horizontal datum is prescribed by function $z(x)$. The fluid layer is $h(x, t)$ deep and moves horizontally with 
velocity $u(x, t)$. The elevation of the fluid surface is $\zeta(x, t)=h(x, t)+z(x)$. Pressure is assumed to be hydrostatic. Under these assumptions the SWE read:

$$
\frac{\partial}{\partial t}\left[\begin{array}{c}
h \\
h u
\end{array}\right]+\frac{\partial}{\partial x}\left[\begin{array}{c}
h u \\
h u^{2}+\frac{1}{2} g h^{2}
\end{array}\right]=\left[\begin{array}{c}
0 \\
-g h \frac{d z}{d x}
\end{array}\right]
$$

and $g$ is the acceleration of gravity. It is clear that the source term is of the form (4), with $G(x)=z(x)$. The SWE present so called equilibrium solutions for fluid at rest whereby the momentum flux and source term cancel each other leading to a flat free surface i.e.: For $u(x, t)=0, \partial h / \partial t=0$ and the second equation gives:

$$
\frac{1}{2} \frac{\partial\left(g h^{2}\right)}{\partial x}(x, t)=-g h \frac{\partial z}{\partial x}(x) \quad \Longleftrightarrow \quad \zeta(x, t)=h(x, t)+z(x)=C
$$

This type of solutions should also be kept at the discrete level.

Definition 5 [11] [12] A numerical scheme for solving (57) verifies the $C$-property if for a stationary equilibrium solution it yields:

$$
h_{i}^{n}+z_{i}=C, \quad \text { and } \quad u_{i}^{n}=0 \quad \forall(i, n) \in \mathbb{Z} \times \mathbb{N},
$$

Proposition 6 Under one of the following two forms of discretisation of the source term at the corrector step:

i) $\left(g h \frac{d z}{d x}\right)_{i}^{n}=\frac{g}{4 \Delta x}\left(h_{i+1 / 2}^{n}+h_{i-1 / 2}^{n}\right)\left(z_{i+1}-z_{i-1}\right)$,

or

ii) $\left(g h \frac{d z}{d x}\right)_{i}^{n}=\frac{g}{8 \Delta x}\left(h_{i+1}^{n}+2 h_{i}^{n}+h_{i-1}^{n}\right)\left(z_{i+1}-z_{i-1}\right)$,

the SRNHS scheme verifies the $C$-property.

PROOF. Let $W(x, t)$ be a stationary solution. Hence $u(x, t)=0$. In such case, system (1) is equivalent to:

$$
\frac{\partial F(W(x, t))}{\partial x}=Q(x, t)
$$

with $W(x, t)=\left[\begin{array}{c}h(x, t) \\ 0\end{array}\right], F=\left[\begin{array}{c}0 \\ \frac{1}{2} g h^{2}(x, t)\end{array}\right]$ and $Q=\left[\begin{array}{c}0 \\ -g h \frac{\partial z}{\partial x}\end{array}\right]$

The eigenvalues of the flux jacobian are:

$$
\overline{\lambda_{1}}=-\bar{c} \quad \text { and } \quad \overline{\lambda_{2}}=\bar{c}
$$


with $\bar{c}^{2}=g \frac{h_{i}^{n}+h_{i+1}^{n}}{2}$ and approximation to the speed of gravity waves at cell interface $x_{i+1 / 2}$. The change matrix $R$ and the inverse change matrix $R^{-1}$ are:

$$
R=\left(\begin{array}{cc}
1 & 1 \\
-\bar{c} & \bar{c}
\end{array}\right) \quad \text { and } \quad R^{-1}=\frac{1}{2 \bar{c}}\left(\begin{array}{cc}
\bar{c} & -1 \\
\bar{c} & 1
\end{array}\right),
$$

According to (17), the predictor step gives:

$$
W_{i+\frac{1}{2}}^{n}=\left[\begin{array}{c}
\frac{h_{i}^{n}+h_{i+1}^{n}}{2} \\
\bar{c}\left(h_{i+1}^{n}+z_{i+1}-h_{i}^{n}-z_{i}\right)
\end{array}\right]=\left[\begin{array}{c}
\frac{h_{i}^{n}+h_{i+1}^{n}}{2} \\
\bar{c}\left(\zeta_{i+1}^{n}-\zeta_{i}^{n}\right)
\end{array}\right]
$$

and the corrector step:

$$
\left[\begin{array}{l}
h_{i}^{n+1} \\
q_{i}^{n+1}
\end{array}\right]=\left[\begin{array}{c}
h_{i}^{n} \\
q_{i}^{n}
\end{array}\right]-\frac{r g}{2}\left[\begin{array}{c}
0 \\
\left(h_{i+1 / 2}^{n}\right)^{2}-\left(h_{i-1 / 2}^{n}\right)^{2}
\end{array}\right]+\Delta t Q_{i}^{n}
$$

In order that the solution remains stationary $W_{i}^{n+1}=W_{i}^{n}$, and the flux and source discretizations must cancel out. This leads to:

$$
\frac{g}{2}\left[\left(h_{i+1 / 2}^{n}\right)^{2}-\left(h_{i-1 / 2}^{n}\right)^{2}\right]=-g\left(h \frac{d z}{d x}\right)_{i}^{n}
$$

or, since $h_{i+1 / 2}=\left(h_{i+1}+h_{i}\right) / 2$, to:

$$
\frac{g}{8 \Delta x}\left(h_{i+1}^{n}+2 h_{i}^{n}+h_{i-1}^{n}\right)\left(h_{i+1}-h_{i-1}\right)=-g\left(h \frac{d z}{d x}\right)_{i}^{n}
$$

but for a stationary solution: $h_{i+1}-h_{i-1}=z_{i-1}-z_{i+1}$. Hence:

$$
\frac{g}{8 \Delta x}\left(h_{i+1}^{n}+2 h_{i}^{n}+h_{i-1}^{n}\right)\left(z_{i+1}-z_{i-1}\right)=g\left(h \frac{d z}{d x}\right)_{i}^{n}
$$

which is equivalent to:

$$
\frac{g}{4 \Delta x}\left(h_{i+1 / 2}^{n}+h_{i-1 / 2}^{n}\right)\left(z_{i+1}-z_{i-1}\right)=g\left(h \frac{d z}{d x}\right)_{i}^{n}
$$

when $u_{i}^{n}=0 \quad \forall i$, what completes the proof.

In order to show the performance of the SRNHS for computing solutions of the SWE, several examples are shown in the following paragraphs. 

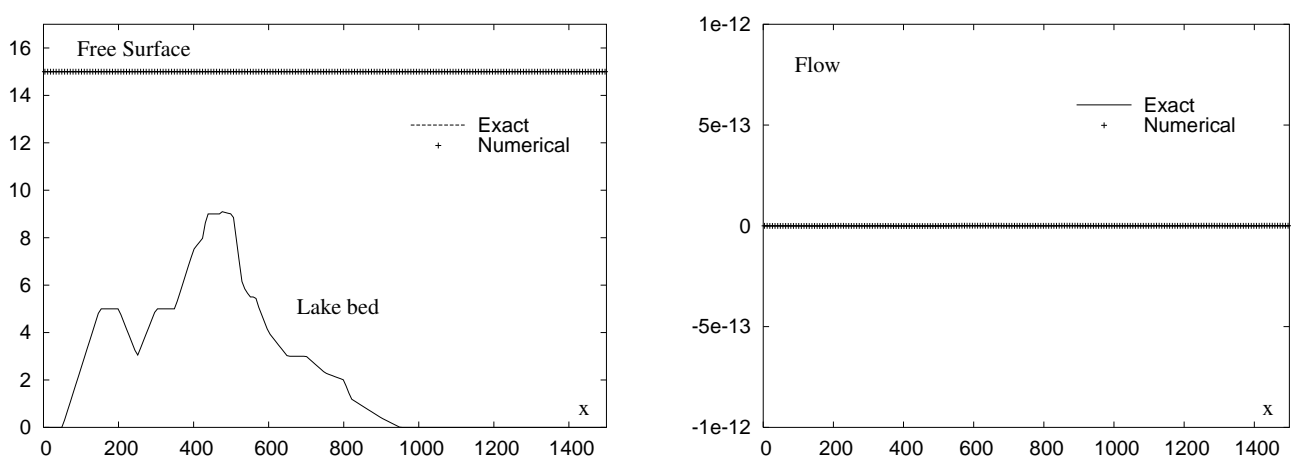

Fig. 3. The lake at rest problem. The free surface of water remains horizontal (left) and the flow rate stays exactly zero (right).

\subsection{The lake at rest problem}

This example was first proposed by [11] to test the $\mathcal{C}$-property compliance of a method. A lake with an irregular bottom is filled with water. The free surface must remain horizontal and water velocity should be zero at all times. Figure 3 shows the computed free surface (left) and velocity (right) after several thousand time steps. As expected the body of water remains exactly at rest.

\subsection{Flow over a bump}

The exact steady state solution shows a constant dicharge $(q=h u)$ but variable free surface and velocity due to the influence of the bed forcing. Depending on the discharge and the inflow and outflow depths, three different flow regimes appear: Subcritical flow all along the reach, transcritical (subcritical to supercritical) without shock and transcritical with shock (smooth subcritical to supercritical, then back to subcritical across a shock). The bump equation used in the following examples is the one given in [60]:

$$
z(x)=\left\{\begin{array}{crr}
0.2-0.05(x-10)^{2}, & \text { if } & 0<x<12 \\
0 & \text { otherwise }
\end{array}\right.
$$

The following examples have been computed on a mesh with 100 cells. Figure 4 shows a comparison between the exact and numerical solution, for the first (subcritical) regime. Left plot displays free surface elevation and right plot Froude number $(F=u / \sqrt{g h})$. The agreement between the numerical and the exact solution can be considered satisfactory. The smooth transcritical regime can be seen in Figure 5. Again satisfactory agreement between the exact and numerical solution is found. The transcritical case with shock is presented in Figure 6 . The shock is well captured with only a small glitch visible in the 

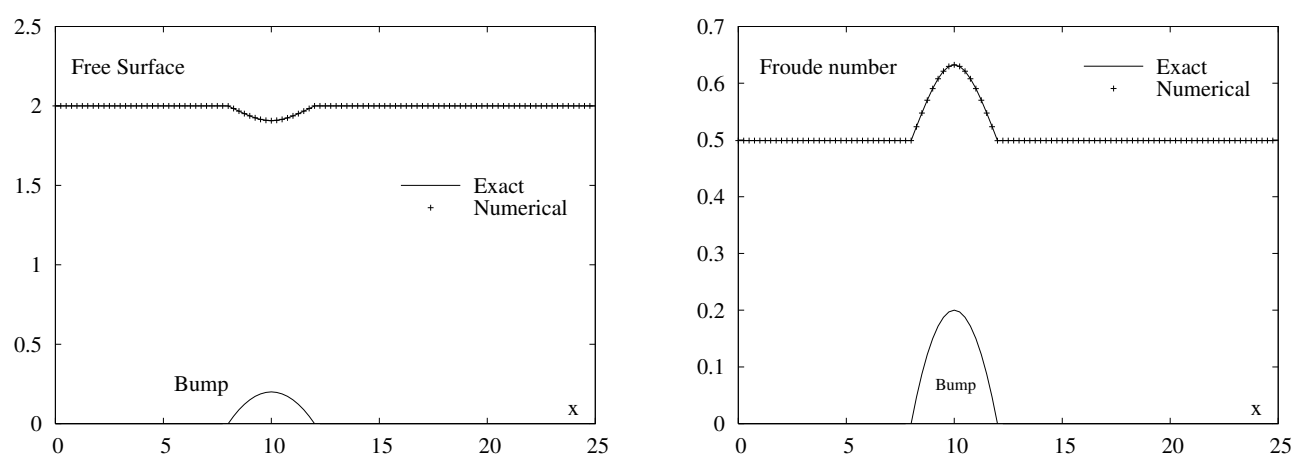

Fig. 4. Comparison between numerical and exact solution for subcritical flow over bump. Free surface (Left) and Froude number (Right).
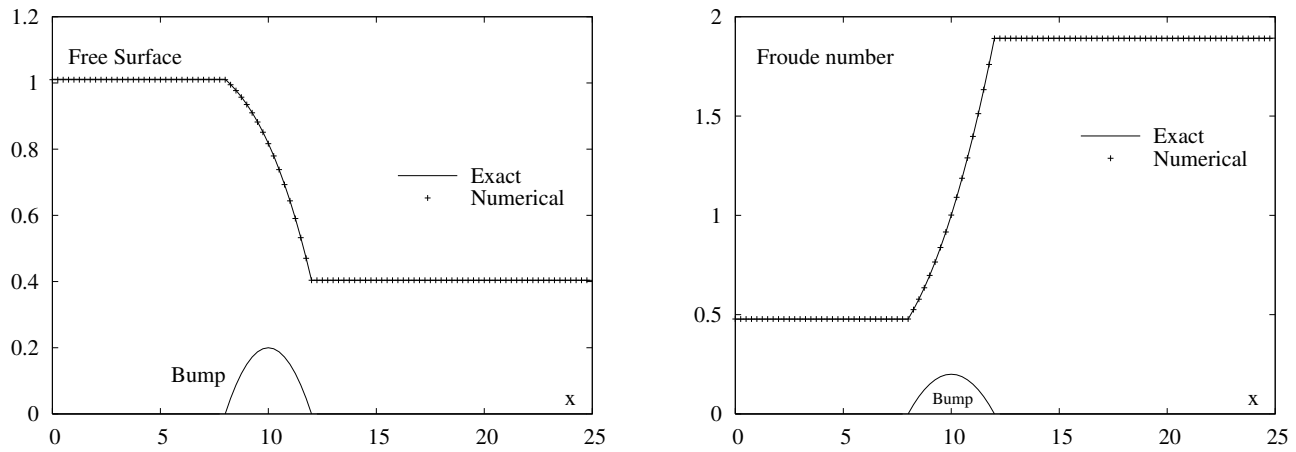

Fig. 5. Transcritical flow over a bump without shock. Free surface (Left) and Froude number (Right).
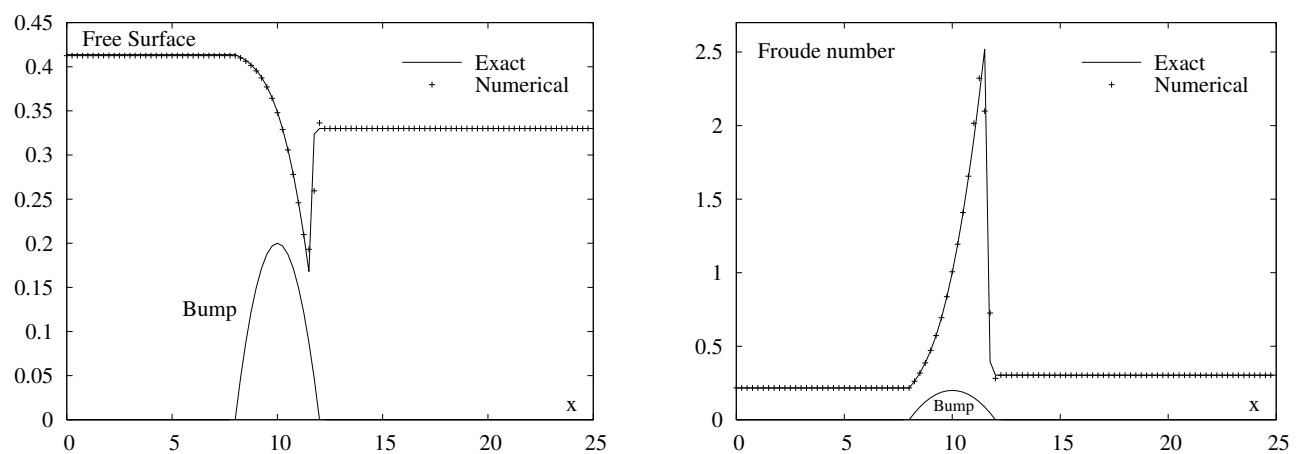

Fig. 6. Transcritical flow over a bump with shock. Free surface (Left) and Froude number (Right).

free surface plot at the downstream end of the shock. The results presented in these examples are comparable to those found in the literature. 
Table 1

Initial data for the dam break over a step problem

\begin{tabular}{|c|c|c|}
\hline & Left & Right \\
\hline$h$ & 5.0 & 1.0 \\
\hline$u$ & 0.0 & 0.0 \\
\hline$z$ & 0.0 & 1.0 \\
\hline
\end{tabular}
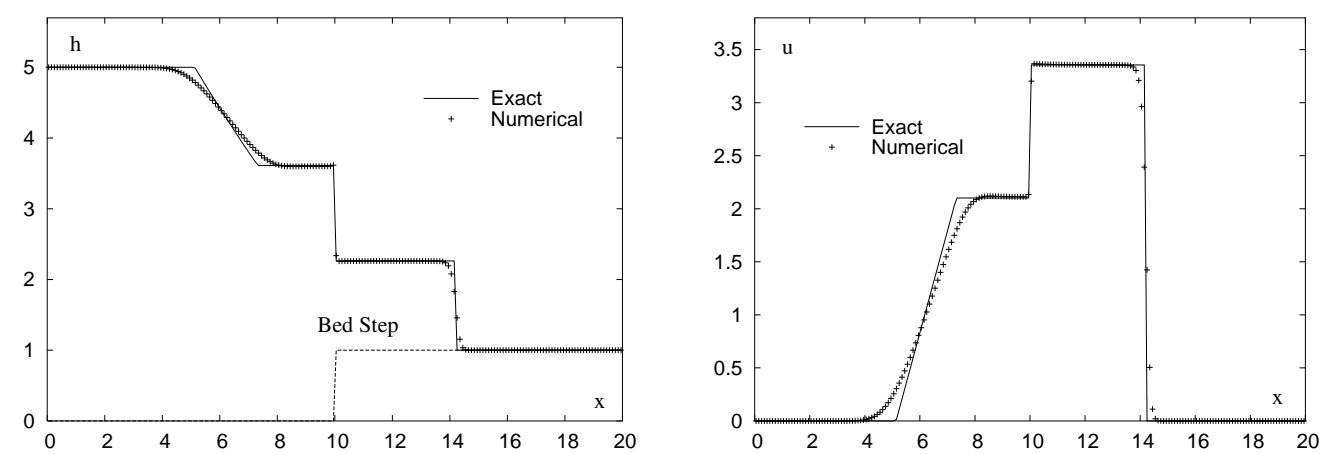

Fig. 7. Dam break problem over a step computed with 200 cells. Water depth (left) and velocity (right) at $\mathrm{t}=0.7 \mathrm{~s} .200$ cells.

\subsection{Dam break flow over a step}

This example corresponds to a Riemann problem with a singular source function. It is hence doubly singular (regarding the initial condition and the source term that becomes a Dirac delta). The particular initial data and bed elevation of the example are displayed in Table 1.

The solution can be exactly computed [3], and for the data above it consists of an expansion wave, a nonlinear discontinuity at the bed step and a travelling shock wave.

Figure 7 shows the numerical solution computed with 200 cells superimposed to the exact one. Left plot corresponds to water depth and right plot to water velocity. All the waves are correctly captured with some smearing of the expansion fan. Figure 8 shows the same comparison for flow rate, $q=h u$, and total head, $H=h+z+u^{2} / 2 g$. These quantities should be constant across the bed step, as it can be realized in the figure, except for a very small perturbation in two points neighbouring the bed discontinuity. The perturbation is more remarkable for the flow rate but almost negligible for the head.

Table 2 provides a comparison of the numeric values of the two constant states that make up the Riemann solution computed exactly and numerically. It can be said that the constant states are sufficiently well captured by the numerical scheme in the 200 cell mesh used. Figure 9 depicts an error convergence plot 

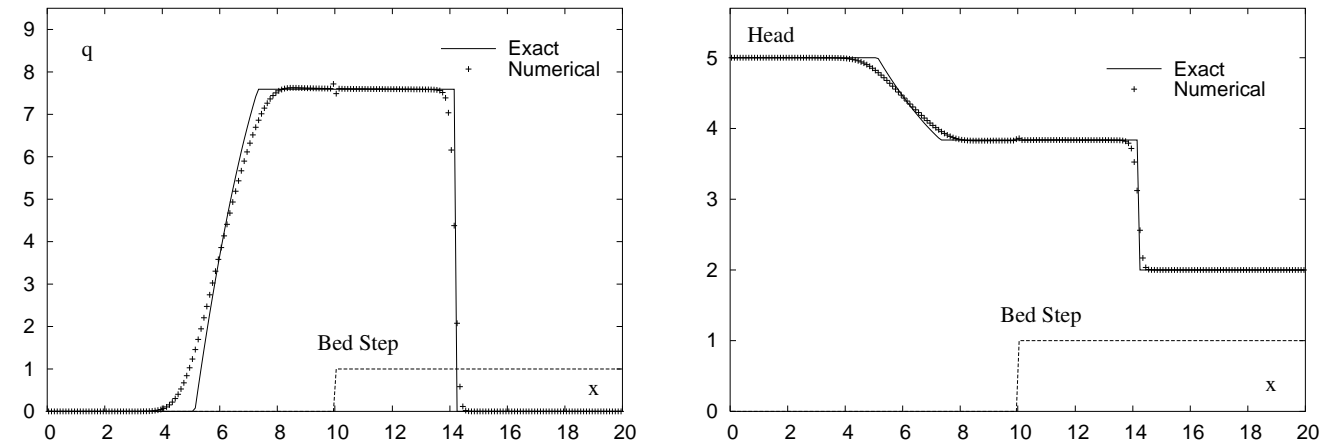

Fig. 8. Dam break problem over a step computed with 200 cells. Flow rate (left) and total head (right) at $\mathrm{t}=0.7 \mathrm{~s}$.

Table 2

Comparison between exact and numerically computed constant states on a 200 cell mesh

\begin{tabular}{|c|c|c|c|c|}
\hline & \multicolumn{2}{|c|}{ Constant State 1 } & \multicolumn{2}{c|}{ Constant State 2 } \\
\hline & Exact & Numerical & Exact & Numerical \\
\hline$h$ & 3.611 & 3.601 & 2.262 & 2.262 \\
\hline$u$ & 2.102 & 2.115 & 3.355 & 3.357 \\
\hline$F r$ & 0.353 & 0.356 & 0.713 & 0.713 \\
\hline
\end{tabular}

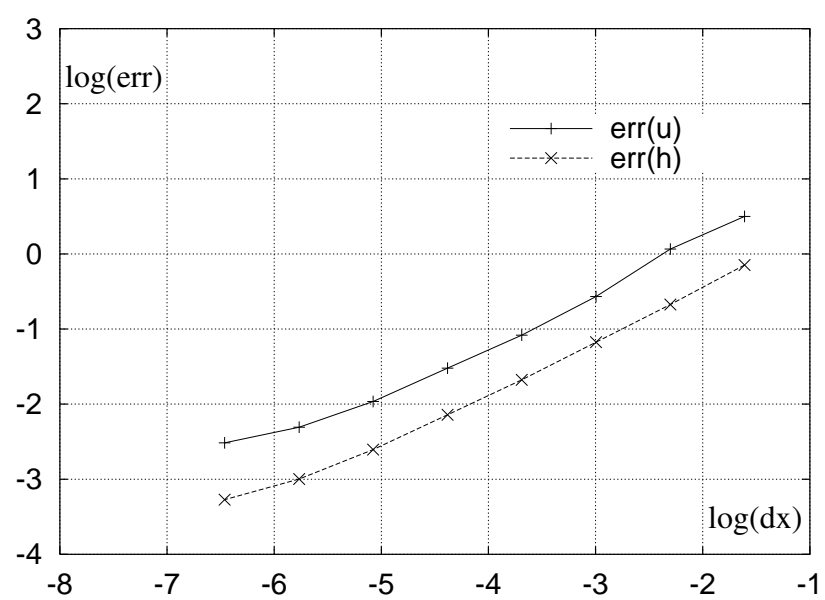

Fig. 9. $L_{1}$ Convergence plot of the velocity and the depth for the dam break over a step.

in the $L_{1}$ norm for the water depth and the velocity. The two curves have an average slope slightly in excess of 0.6 . However this value shows a tendency to decrease as the mesh is refined, eventually leading to a stagnation of the convergence. This undesirable behaviour is problem dependent and manifests itself more strongly for some initial data than for others. The stagnation tendency appears also for other systems of equations with singular source terms as will be shown later for the Euler equations. Furthermore, most finite volume 

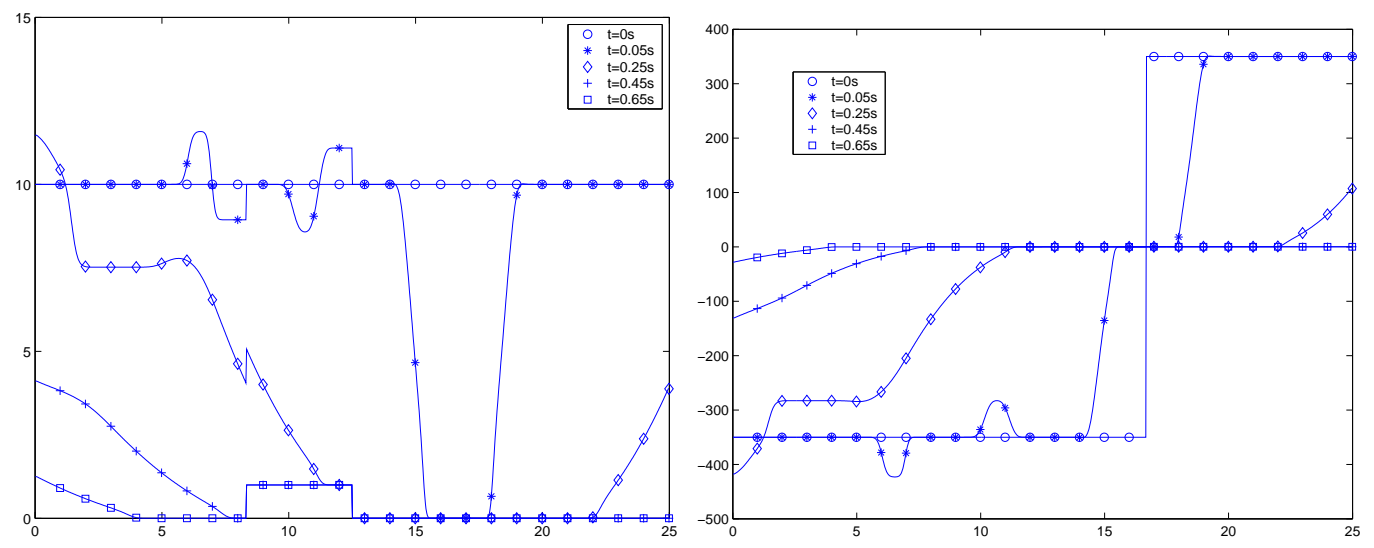

Fig. 10. Double rarefaction and dry out of a step. Free surface elevation (left) and flow rate (rifgt) at $t=1 \mathrm{~s}$. The step geometry can be seen in the left plot centred around $\mathrm{x}=11 \mathrm{~m}$.

methods can suffer from this behaviour. An explanation of this phenomenon together with a possible cure is discussed in section 9 .

\subsection{Dry out by a double rarefaction over a step}

In this test, first proposed in [24] the combined ability of the method to deal with a step bottom function and dry zones $(h=0)$ is shown. Two rarefaction fans travelling in opposite directions create a dry zone or vacuum. The left running rarefaction attains and passes over a stepped bed elevation. Here the exact solution is not available, but results shown in Figure 10 compare well with those presented in the original reference.

\section{Application to the non-isentropic Euler equations in a duct of variable cross section}

In this paragraph the quasi one dimensional, unsteady, compressible flow of a gas along a nozzle with variable cross section $A(x)$ is considered. The governing (Euler) equations can be written:

$$
\frac{\partial}{\partial t}\left[\begin{array}{c}
\rho A \\
\rho A u \\
\rho A E
\end{array}\right]+\frac{\partial}{\partial x}\left[\begin{array}{c}
\rho A u \\
\rho A\left(u^{2}+p / \rho\right) \\
\rho A u H
\end{array}\right]=\left[\begin{array}{c}
0 \\
p \frac{d A}{d x} \\
0
\end{array}\right]
$$


where $\rho, u$ and $p$ are the gas density, velocity and pressure respectively. $E$ and $H$ represent the total energy and total enthalpy:

$$
E=\frac{p}{(\gamma-1) \rho}+\frac{u^{2}}{2} \quad H=\frac{\gamma p}{(\gamma-1) \rho}+\frac{u^{2}}{2}
$$

with $\gamma$ the ratio of specific heats (in all numerical examples $\gamma$ has been taken as 1.4) and the perfect gas law applies. By calling the conservative variables $m_{1}=\rho A, m_{2}=\rho A u$, and $m_{3}=\rho A E$, system (70) can be written:

$$
\begin{gathered}
\frac{\partial}{\partial t}\left[\begin{array}{l}
m_{1} \\
m_{2} \\
m_{3}
\end{array}\right]+\frac{\partial}{\partial x}\left[\begin{array}{c}
m_{2} \\
\frac{(3-\gamma) m_{2}^{2}}{2 m_{1}}+(\gamma-1) m_{3} \\
\frac{(1-\gamma) m_{2}^{2}}{2 m_{1}}+\gamma m_{3}
\end{array}\right] \\
=\left[\begin{array}{c}
\left(\frac{(1-\gamma) m_{2}^{2}}{2 m_{1}^{2}}+\frac{(\gamma-1) m_{3}}{2 A}\right) \frac{d A}{d x} \\
0
\end{array}\right]
\end{gathered}
$$

which is clearly in the form (1) with source term (4).

The SRNHS can be applied straightforwardly to system (72). The source term discretization at the predictor step can be performed as:

$$
\left(p \frac{d A}{d x}\right)_{i+1 / 2}^{n}=\frac{p_{i+1}+p_{i}}{2 \Delta x}\left(A_{i+1}-A_{i}\right)
$$

And for the corrector step:

$$
\left(p \frac{d A}{d x}\right)_{i}^{n}=\frac{p_{i+1}+2 p_{i}+p_{i-1}}{4} \frac{\left(A_{i+1}-A_{i-1}\right)}{2 \Delta x}
$$

although other choices are possible.

As an example of the performance of the method, the computation of a shock tube problem with discontinuous cross section is shown. This test corresponds also to a Riemann problem with a singular source term and the exact solution can also be computed analytically (see [5]). The initial data are displayed in Table 3 and the discontinuity is located at $x=5$. For this case, the solution consists of a left running rarefaction, a stationary discontinuity at $x=5$ due to the cross section area change, a right running contact and a shock.

Figure 11 shows a comparison between the exact and the numerical solution for density (left) and Mach number (right). The cross section is also shown 
Table 3

Initial data for the shock tube problem

\begin{tabular}{|c|c|c|}
\hline & Left & Right \\
\hline$\rho$ & 2.0 & 1.0 \\
\hline$u$ & 0.0 & 0.0 \\
\hline$p$ & 6.0 & 1.0 \\
\hline$A$ & 0.15 & 0.1 \\
\hline
\end{tabular}
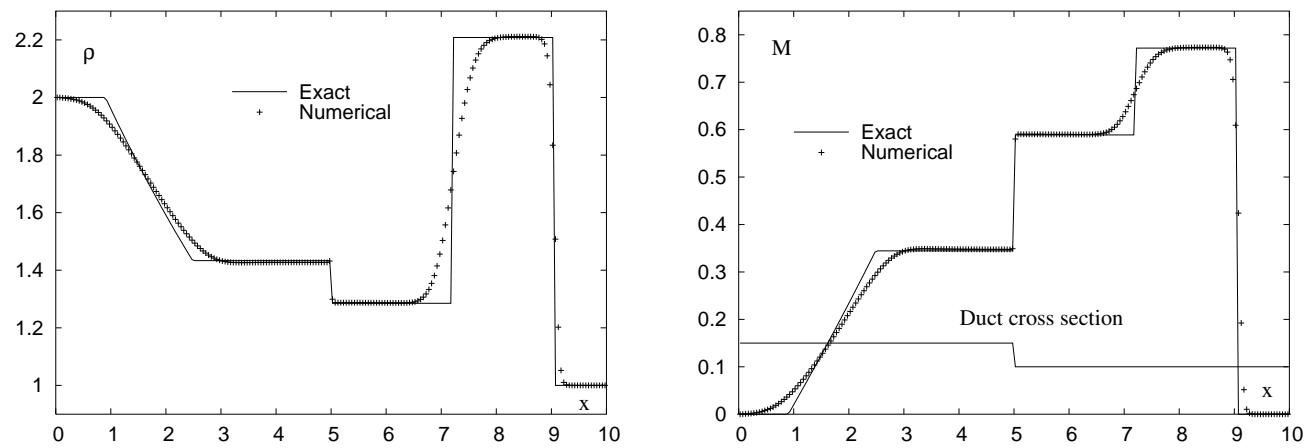

Fig. 11. Shock tube problem with discontinuous cross section computed with 200 cells. Density (left) and Mach number (right) at $\mathrm{t}=2 \mathrm{~s}$ (the duct cross section area is plotted also as a dotted line in the Mach plot).

as a solid line in the Mach plot. Figure 12 corresponds to the same comparison for the entropy (left) and the mass flow (right) that should be constant across the duct cross section discontinuity. The performance of the scheme is directly related to its ability to capture the constant states to both sides of the cross section discontinuity as well as to resolve the different waves: Rarefaction, stationary discontinuity, moving contact and shock. Overall the numerical solution computed with 200 nodes shows good agreement with the exact one. It is worth noting that the $S R N H S$ scheme captures sharply the stationary contact with no intermediate points in this particular case. The right running contact is a linear wave and hence it is diffused. Also the numerical mass flow and entropy remain constant across the cross section discontinuity as the theory predicts. Table 7 shows a comparison of the numerical versus exactly computed constant intermediate states. The agreement can be considered good.

Figure 13 displays a convergence plot in the $L_{1}$ norm for the gas density and velocity. The average slope is slightly below 0.5 for the density and slighlty above 0.4 for the velocity. In this case the convergence decay is more clearly visible than for the SWE test (Figure 9), with both curves rapidly approaching a stagnation condition. This phenomenon is analyzed in section 9 . 

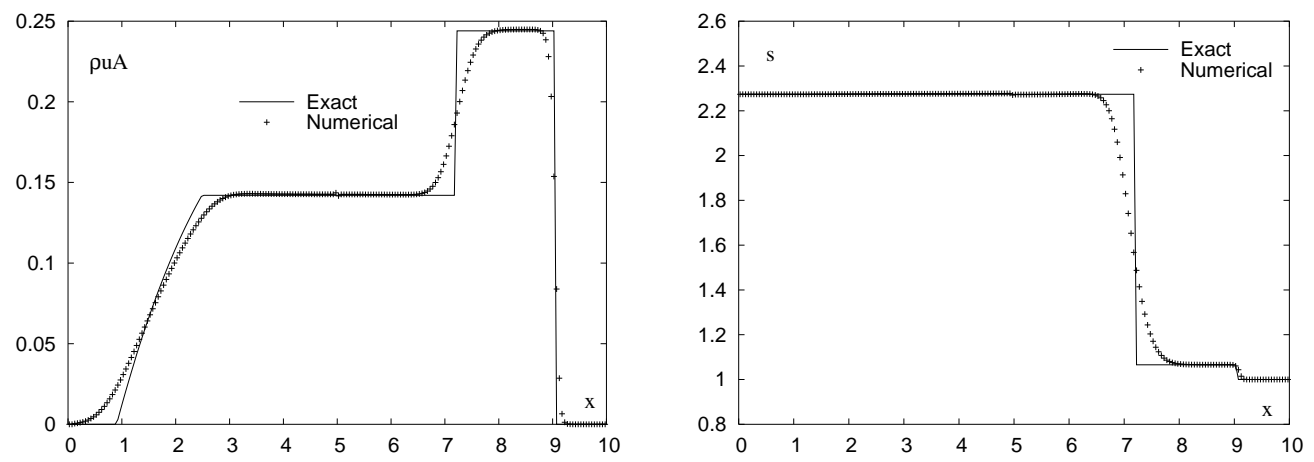

Fig. 12. Shock tube problem with discontinuous cross section computed with 200 cells. Mass flow (left) and entropy (right) at $\mathrm{t}=2 \mathrm{~s}$. Both quantities must be constant across the cross section discontinuity.

Table 4

Comparison between exact and numerically computed constant states on a 200 cell mesh

\begin{tabular}{|c|c|c|c|c|c|c|}
\hline & \multicolumn{2}{|c|}{ Constant State 1 } & \multicolumn{2}{c|}{ Constant State 2 } & \multicolumn{2}{c|}{ Constant State 3 } \\
\hline & Exact & Numerical & Exact & Numerical & Exact & Numerical \\
\hline$\rho$ & 1.433 & 1.427 & 1.285 & 1.287 & 2.208 & 2.211 \\
\hline$u$ & 0.661 & 0.666 & 1.105 & 1.107 & 1.105 & 1.107 \\
\hline$p$ & 3.764 & 3.747 & 3.231 & 3.237 & 3.231 & 3.238 \\
\hline$M$ & 0.345 & 0.347 & 0.589 & 0.590 & 0.772 & 0.773 \\
\hline
\end{tabular}

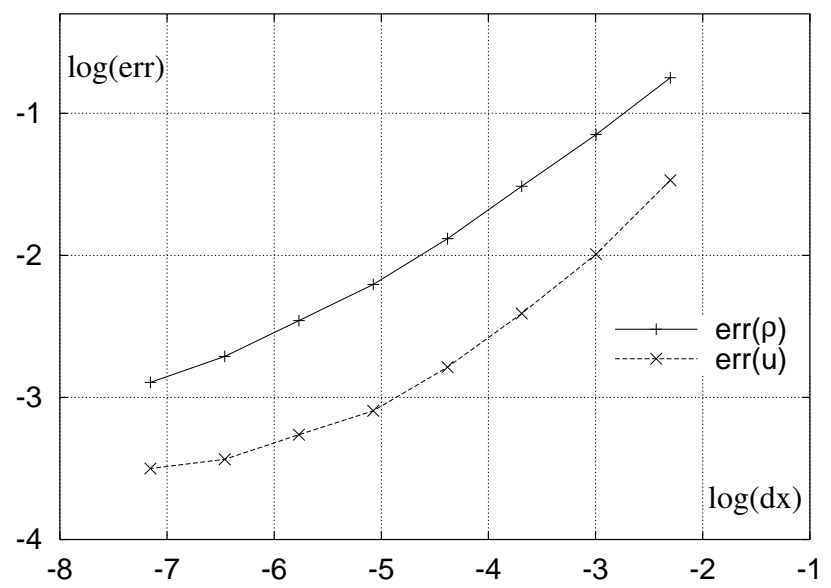

Fig. 13. $L_{1}$ Convergence plot of the density and velocity for the shock tube problem with cross section discontinuity. 


\section{Application of the $S R N H S$ scheme to a multiphase flow model problem: The Ransom faucet problem}

This section is devoted to the extension of the SRNHS scheme to biphasic (liquid-gas) flow systems by means of a four equation single pression model. The system can be written as (1):

$$
\frac{\partial W}{\partial t}+\frac{\partial F(W)}{\partial x}=Q_{1}+Q_{2}
$$

with conservative variables, $W$, and flux vector $F$ :

$$
W(x, t)=\left(\begin{array}{c}
\alpha_{v} \rho_{v} \\
\alpha_{v} \rho_{v} u_{v} \\
\alpha_{l} \rho_{l} \\
\alpha_{l} \rho_{l} u_{l}
\end{array}\right), \quad F(W(x, t))=\left(\begin{array}{c}
\alpha_{v} \rho_{v} u_{v} \\
\alpha_{v} \rho_{v} u_{v}^{2} \\
\alpha_{l} \rho_{l} u_{l} \\
\alpha_{l} \rho_{l} u_{l}^{2}
\end{array}\right)
$$

$\alpha, \rho$ and $u$ represent the mass fraction, density and velocity respectively of the vapour (subindex $v$ ) or the liquid (subindex $l$ ). The source term $Q(W)$ is split into two parts, $Q_{1}$ and $Q_{2}$. $Q_{1}$ accounts for the interchange of momentum between both phases and $Q_{2}$ represents just the effect of the acceleration due to gravity $(g)$ :

$$
Q_{1}(W)=-\left(\begin{array}{c}
0 \\
\alpha_{v} \frac{\partial p}{\partial x} \\
0 \\
\alpha_{l} \frac{\partial p}{\partial x}
\end{array}\right), \quad Q_{2}(W)=\left(\begin{array}{c}
0 \\
\alpha_{v} \rho_{v} g \\
0 \\
\alpha_{l} \rho_{l} g
\end{array}\right)
$$

The pressure, $p$, is common to both phases. In order to close the system the following relations apply: $\alpha_{v}+\alpha_{l}=1$. The isentropic equation of state for the gas phase, $p=c \rho_{v}^{\gamma}$, and a equation of state for the liquid: $\rho_{l}=k_{l} p^{a}$. Here $C$, $\gamma, a$ and $k_{l}$ are constants. Typical numerical values (in International System units) are: $C=10^{5}, \gamma=1.4, a=4.37 \times 10^{-5}$ and $k_{l}=987,57$.

The system represented by equations (76) and (77) is not hyperbolic. Therefore the flux jacobian $\nabla F(W)$ can not be diagonalised in $\mathbb{R}$ what makes it difficult the computation of a sign matrix as required by the $S R N H S$ scheme. In order to overcome this difficulty the system is written as follows:

$$
\frac{\partial W(x, t)}{\partial t}+\nabla F(W(x, t)) \frac{\partial W(x, t)}{\partial x}+C(W) \frac{\partial W(x, t)}{\partial x}=Q_{2}(x, W)
$$


with $C(W) \frac{\partial W(x, t)}{\partial x}=-Q_{1}(x, W)$.

The source term $Q_{2}$ has not the form (4) and hence does not fit within the framework for which $S R N H S$ scheme is developed. For this reason equation (78) is integrated in two steps. The first step gets rid of the weight term, $Q_{2}$, by an explicit Euler time integration:

$$
\left\{\begin{array}{l}
\frac{\partial \hat{W}}{\partial t}=Q_{2}(\hat{W}) \\
\hat{W}\left(x, t^{n}\right)=W^{n}(x)
\end{array}\right.
$$

and then the SRNHS scheme is applied to the system:

$$
\left\{\begin{array}{l}
\frac{\partial W(x, t)}{\partial t}+\frac{\partial F(W(x, t))}{\partial x}+Q_{1}(x, W)=0 \\
W\left(x, t^{n}\right)=\hat{W}^{n+1}(x)
\end{array}\right.
$$

Taking $A(W)=\nabla F(W(x, t))+C(W)$, the first equation of (80) can be written in quasilinear form:

$$
\frac{\partial W(x, t)}{\partial t}+A(W) \frac{\partial W(x, t)}{\partial x}=0
$$

with

$$
A(\bar{W})=\left(\begin{array}{cccc}
0 & 1 & 0 & 0 \\
-\bar{u}_{v}^{2}+\frac{\gamma \bar{p}}{\rho_{v}} 2 \bar{u}_{v} & \frac{\gamma \bar{p}}{\bar{\rho}_{l}} & 0 \\
0 & 0 & 0 & 1 \\
\frac{\bar{\alpha}_{l}}{\bar{\alpha}_{v}} \frac{\gamma \bar{p}}{\bar{\rho}_{v}} & 0 & -\bar{u}_{l}^{2}+\frac{\bar{\alpha}_{l}}{\bar{\alpha}_{v}} \frac{\gamma \bar{p}}{\bar{\rho}_{l}} & 2 \bar{u}_{l}
\end{array}\right) .
$$

The predictor step of $S R N H S$ scheme is applied to the system (80) written in the form (81) as follows:

$$
W_{i+\frac{1}{2}}^{n}=\frac{1}{2}\left(W_{i}^{n}+W_{i+1}^{n}\right)-\frac{1}{2} \operatorname{sgn}(A(\bar{W}))\left(W_{i+1}^{n}-W_{i}^{n}\right)
$$

with $\bar{W}=\left(W_{i+1}^{n}+W_{i}^{n}\right) / 2$. The corrector step is simply:

$$
W_{i}^{n+1}=W_{i}^{n}-r\left(F\left(W_{i+\frac{1}{2}}^{n}\right)-F\left(W_{i-\frac{1}{2}}^{n}\right)\right)+\Delta t\left(Q_{1}\right)_{i}^{n}
$$


with the following approximation of $\left(Q_{1}\right)_{i}^{n}$ :

$$
\left(Q_{1}\right)_{i}^{n}=\frac{1}{2 \Delta x}\left[\begin{array}{c}
0 \\
\left(\alpha_{v}\right)_{i}^{n}\left(p_{i+1}^{n}-p_{i-1}^{n}\right) \\
0 \\
\left(\alpha_{l}\right)_{i}^{n}\left(p_{i+1}^{n}-p_{i-1}^{n}\right)
\end{array}\right]
$$

Keeping in mind that the first step of SRNHS scheme is essentially an upwinding of the variables to compute $W_{j+1 / 2}^{n}$, the only difficulty lies in the calculation of the $\operatorname{sgn}(A)$ matrix because, as stated before, for this problem it is not diagonalizable and its eigenvalues are complex.

In this work the method of Alouges [7] has been used to compute the real part of the eigenvalues of $A$ and from these an approximate sign matrix. The predictor step of $S R N H S$ scheme has been applied making use of the approximate sign matrix.

\section{Density perturbation method}

Another way to overcome the problem of the calculation of the sign matrix for multiphase flows is the method proposed by Toumi and his co-authors [18] based upon an extension of the domain of hyperbolicity of system (76)-(77). This is accomplished by means of a modifiction of the term $Q_{1}$, that becomes:

$$
Q_{1}(W)=\left[\begin{array}{c}
0 \\
\alpha_{v} \frac{\partial p}{\partial x}+\delta\left(p-p_{v}^{i n t}\right) \frac{\partial \alpha_{v}}{\partial x} \\
0 \\
\alpha_{l} \frac{\partial p}{\partial x}+\delta\left(p-p_{l}^{i n t}\right) \frac{\partial \alpha_{l}}{\partial x}
\end{array}\right]
$$

where formula:

$$
\delta\left(p-p_{k}^{i n t}\right) \frac{\partial \alpha_{k}}{\partial x}
$$

accounts for a pressure correction, and $p_{k}^{\text {int }}$ is the interfacial pressure between both phases with $\delta$ a numeric constant. There are several forms of the pressure difference $\left(p-p_{k}^{\text {int }}\right)$ in the literature. Here we adopt the closure law for bubbly 
flow given by Lahey [42]:

$$
\left\{\begin{array}{l}
p-p_{v}^{i n t}=0 \\
p-p_{l}^{\text {int }}=C_{p}\left(\alpha_{v}\right) \rho_{l}\left(u_{v}-u_{l}\right)^{2}
\end{array}\right.
$$

where $C_{p}\left(\alpha_{v}\right)=\frac{1}{4}\left(1-\alpha_{v}\right)$ for instance.

In what follows we will call: $\theta_{l}=\delta\left(p-p_{l}^{\text {int }}\right)$

In order to calculate $\operatorname{sgn}(A)$ a perturbation analysis based on the density is performed [18]. The dimensionless density of each phase is defined as: $\tilde{\rho}_{v}=\frac{\rho_{v}}{\rho_{v}^{0}}$ and $\tilde{\rho}_{l}=\frac{\rho_{l}}{\rho_{l}^{0}}$. With $\rho_{v}^{0}$ and $\rho_{l}^{0}$ a reference density of the gas and liquid phase respectively. Let the parameter $\epsilon=\frac{\rho_{v}^{0}}{\rho_{l}^{0}}$, with $\epsilon<<1$ in view of the density difference between the liquid and vapour phases. Further assume that the liquid phase is incompressible, i.e.: $\rho_{l}=\rho_{l}^{0}$ or $\tilde{\rho}_{l}=1$.

Now system (80) is written again in quasilinear form (81), with $A(W)$ :

$$
A(W)=\left(\begin{array}{cccc}
0 & 1 & 0 & 0 \\
-u_{v}^{2}+\alpha_{v} p_{, 1} & 2 u_{v} & \alpha_{v} p_{, 3} & 0 \\
0 & 0 & 0 & 1 \\
\epsilon \alpha_{l} p_{, 1}+\theta_{l} \alpha_{l, 1} & 0 & -u_{l}^{2}+\epsilon \alpha_{l} p_{, 3}+\theta_{l} \alpha_{l, 3} & 2 u_{l}
\end{array}\right)
$$

In such case following [Marc 98], [26] one has: $\alpha_{v} p_{, 1}=\frac{\gamma p}{\rho_{v}}, p_{, 3}=\frac{\gamma p}{\alpha_{v} \rho_{l}}$, since $\rho_{l}=\rho_{l}^{0}$ is constant one has $p_{, 3}=\frac{\gamma p}{\alpha_{v} \rho_{v}^{0}} \frac{\rho_{v}^{0}}{\rho_{l}^{0}}=\epsilon \frac{\gamma p}{\alpha_{v} \rho_{v}^{0}}, \alpha_{l, 1}=0$ and $\alpha_{l, 3}=\frac{1}{\rho_{l}}$.

Calling

$$
\frac{\gamma p}{\rho_{v}}=c_{1}^{2} \quad \text { and } \quad c_{2}^{2}=\frac{\theta_{l}}{\rho_{l}}
$$

and going back to (89) one can write: $A(W)=A_{0}(W)+\epsilon H(W)$ with

$$
A_{0}(W)=\left(\begin{array}{cccc}
0 & 1 & 0 & 0 \\
-u_{v}^{2}+c_{1}^{2} & 2 u_{v} & 0 & 0 \\
0 & 0 & 0 & 1 \\
0 & 0 & -u_{l}^{2}+c_{2}^{2} & 2 u_{l}
\end{array}\right)
$$


and

$$
H(W)=\left(\begin{array}{cccc}
0 & 0 & 0 & 0 \\
0 & 0 & \frac{\gamma p}{\rho_{v}^{0}} & 0 \\
0 & 0 & 0 & 0 \\
\alpha_{l} p_{, 1} & 0 & \alpha_{l} p_{, 3} & 0
\end{array}\right)
$$

$A_{0}(W)$ can be diagonalized. Its characteristic polynome is:

$$
P_{0}(\lambda)=\left(\left(\lambda-u_{v}\right)^{2}-c_{1}^{2}\right)\left(\left(\lambda-u_{l}\right)^{2}-c_{2}^{2}\right)
$$

that admits four distinct real eigenvalues:

$$
\lambda_{1}=u_{v}-c_{1}, \quad \lambda_{2}=u_{v}+c_{1}, \quad \lambda_{3}=u_{l}-c_{2}, \quad \lambda_{4}=u_{l}+c_{2}
$$

The corresponding right eigenvector or change matrix, $R$, is:

$$
R(W)=\left(\begin{array}{cccc}
1 & 1 & 0 & 0 \\
\lambda_{1} & \lambda_{2} & 0 & 0 \\
0 & 0 & 1 & 1 \\
0 & 0 & \lambda_{3} & \lambda_{4}
\end{array}\right)
$$

The perturbation theory of linear operators $[35,14,38]$ states that if a linear operator $B$ is diagonisable in $\mathbb{R}$ with distinct eigenvalues and it is perturbed to $B^{\prime}=B+\epsilon H$ with $\epsilon$ a small real number, then $B^{\prime}$ can be also diagonalised in $\mathbb{R}$ with distinct eigenvalues, these are close to the eigenvalues of $B$ and the following relation holds:

$$
\left|\lambda_{j}^{\prime}-\lambda_{j}\right|=\circ(\epsilon)
$$

With this result in mind it is justifiable to apply $S R N H S$ scheme to solve system (80) as follows:

$$
\left\{\begin{array}{l}
W_{i+\frac{1}{2}}^{n}=\frac{1}{2}\left(W_{i}^{n}+W_{i+1}^{n}\right)-\frac{1}{2} \operatorname{sgn}\left(A_{0}(\bar{W})\right)\left(W_{i+1}^{n}-W_{i}^{n}\right) \\
W_{i}^{n+1}=W_{i}^{n}-r\left(F\left(W_{i+\frac{1}{2}}^{n}\right)-F\left(W_{i-\frac{1}{2}}^{n}\right)\right)+\Delta t\left(S_{1}\right)_{i}^{n},
\end{array}\right.
$$


with:

$$
\left(Q_{1}\right)_{i}^{n}=\left[\begin{array}{c}
0 \\
\frac{\left(\alpha_{v}\right)_{i}^{n}}{2 \Delta x}\left(p_{i+1}^{n}-p_{i-1}^{n}\right) \\
0 \\
\frac{\left(\alpha_{l}\right)_{i}^{n}}{2 \Delta x}\left(p_{i+1}^{n}-p_{i-1}^{n}\right)-\frac{1}{2 \Delta x} \delta\left(\rho_{l}\right)_{i}^{n}\left(\alpha_{v}\right)_{i}^{n}\left(\left(u_{l}\right)_{i}^{n}-\left(u_{v}\right)_{i}^{n}\right)^{2}\left(\left(\alpha_{l}\right)_{i+1}^{n}-\left(\alpha_{l}\right)_{i-1}^{n}\right)
\end{array}\right]
$$

\subsection{Ransom faucet tests}

The performance of $S R N H S$ scheme for solving system (75) is shown in the following examples computed. They regard the so called Ransom faucet problem [49] in which a water jet exits a faucet downwards in the vertical direction surrounded by standing air. The initial condition corresponds to a cylindrical jet. A sketch is shown in Figure 14. The liquid volume fraction, $\alpha_{l}$, is in this case the ratio of the jet section to the total cross section and the void fraction, $\alpha_{v}$, the ratio of the cross section occupied by the air to the total cross section. By the effect of gravity the jet accelerates while narrowing its cross section. This corresponds to a wave of void fraction propagating downstream. (The hypothesis is made here that the jet does not break up in drops).

The initial and boundary conditions corresponding to the Ransom faucet test are given in Figure 14 and Table 5:

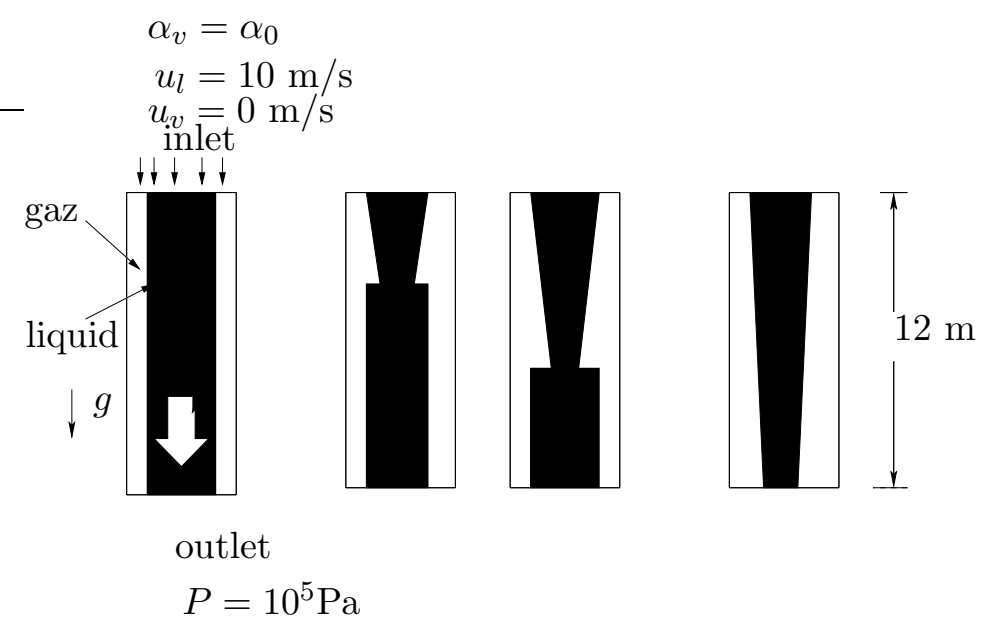

Fig. 14. Sketch of the Ransom faucet problem.

Two tests are shown, one for a low initial void fraction $\left(\alpha_{v}(t=0)=0.2\right)$ and other for a larger one $\left(\alpha_{v}(t=0)=0.6\right)$. It proves more difficult to compute 
Table 5

Initial and boundary conditions for Ransom faucet problem:

\begin{tabular}{|l||l|}
\hline Initial condition: & Boundary conditions: \\
\hline \hline$\forall x \in\left[x_{0}, x_{l}\right], \alpha_{v}(t=0)=\alpha_{0}$, & * inlet $\left(x_{0}=0\right): \alpha_{v}(0, t)=\alpha_{0}$, \\
\hline$u_{l}(t=0)=10, u_{v}(t=0)=0$, & $* \operatorname{inlet}\left(x_{0}=0\right): u_{l}(0, t)=10$, \\
\hline$\rho_{v}(t=0)=1, \rho_{l}(t=0)=988,0638$, & $* \operatorname{inlet}\left(x_{0}=0\right): u_{v}(0, t)=0$, \\
\hline$p(t=0)=10^{5}$. & $*$ outlet $\left(x_{l}=12\right): p(12, t)=10^{5}$. \\
\hline
\end{tabular}
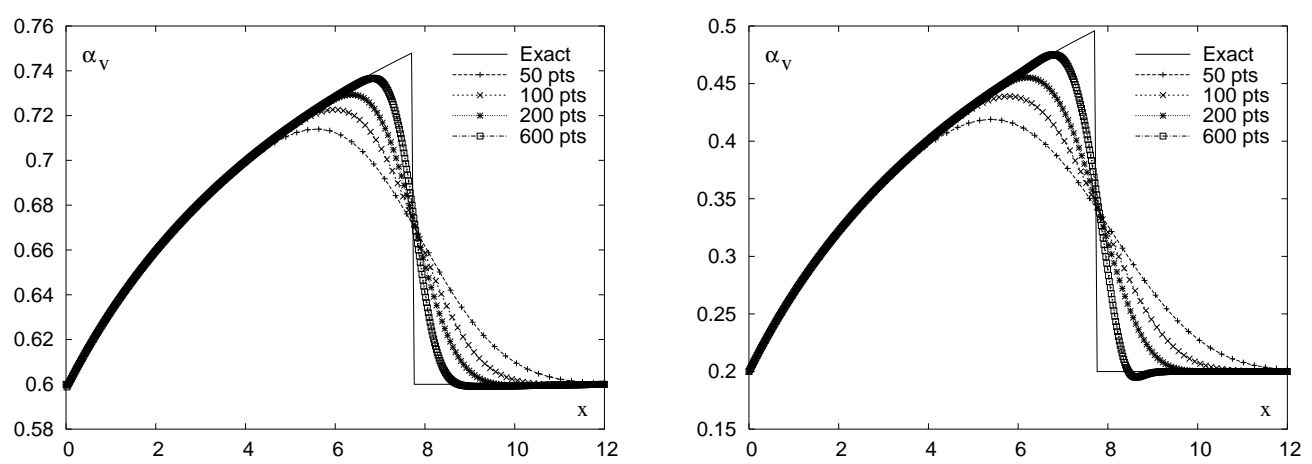

Fig. 15. Void fraction at $\mathrm{t}=0.6 \mathrm{~s}$ for initial value of 0.6 (left) and 0.2 (right) computed with $S R N H S$ scheme on a sequence of meshes.

flows with lower void fractions.

The numerical results are compared to the exact solution $W_{e}$ of an analogous but well posed problem, obtained supposing the liquid and vapour densities are constant. The reference solution is given by:

$$
\alpha_{v, e}(x, t)= \begin{cases}1-\frac{\alpha_{l, \text { init }} u_{l, \text { init }}}{\sqrt{2 g x+u_{l, \text { init }}^{2}}} & \text { if } \quad x \leq u_{l, \text { init }} t+\frac{g t^{2}}{2} \\ 1-\alpha_{v, \text { init }} & \text {, otherwise }\end{cases}
$$

and

$$
u_{l, e}(x, t)= \begin{cases}\sqrt{2 g x+u_{l, \text { init }}^{2}} & \text { if } \quad x \leq u_{l, \text { init }} t+\frac{g t^{2}}{2} \\ u_{l, \text { init }}+g t & \text {, otherwise }\end{cases}
$$

Figure 15 shows the void fraction, $\alpha_{v}$, computed with different mesh sizes (from 50 to 600 nodes) at time $t=0.6 \mathrm{~s}$ for two initial values of the void fraction $\alpha_{v}(0)$.

The left plot corresponds to $\alpha_{v}(0)=0.6$ and the right plot to $\alpha_{v}(0)=0.2$. The former case has been computed with the method of Alouges for the calculation of the sign matrix in the predictor step. The second with the regularization method of Toumi as explained ealier. The advantage of the method of Toumi 

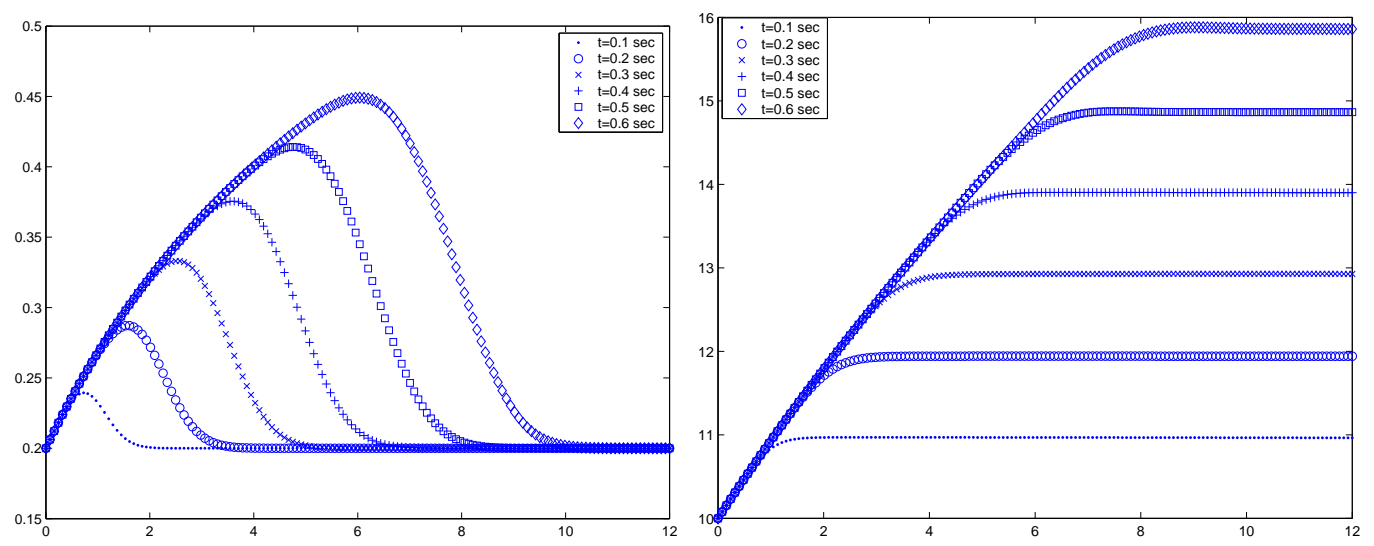

Fig. 16. Time evolution of the void fraction (left) and the liquid velocity (right) for an initial void fraction of 0.6

lies in that it proves more robust despite the need for the adjustable hyperbolicity parameter $\delta$ (equation 86). Actually, the case $\alpha_{v}(0)=0.6$ can not be computed with the method of Alouges due to numerical instabilites. Both plots in figure 15 show a reasonable agreement with the exact solution that improves with the mesh size and are comparable to those presented by other authors. Nevertheless mid sized meshes (around 200 nodes) provide a sufficiently accurate description of the phenomenon.

Figure 16 shows the evolution of $\alpha_{v}$ (left) and the liquid velocity $u_{l}$ (right) with time. The initial conditions correspond to $\alpha_{v}(0)=0.6$ and the method of Toumi has been used with $\delta=5 \cdot 10^{-4}$. The propagation of the front is well predicted numerically with 200 nodes.

\section{The convergence stagnation problem}

This section is devoted to give an explanation to the convergence stagnation phenomenon observed in the previous sections. We will show that for a linear scalar equations with source terms of the form (4) with discontinuities in the function $G(x, W)$, convergence of the numerical to the exact solution is prevented. This gives an indication that a similar behaviour can be found for nonlinear systems, as it has been indeed observed in previous examples. A cure is also proposed that solves this problem from the theoretical point of view. Let us consider the scalar equation:

$$
\frac{\partial u}{\partial t}+a \frac{\partial u}{\partial x}=-u \frac{d z}{d x}
$$




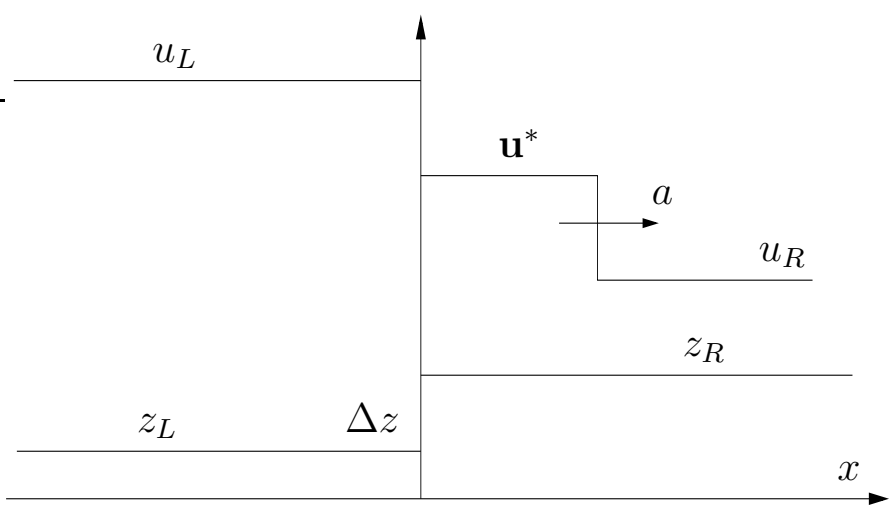

Fig. 17. The Riemann solution for the linear equation.

with $a>0$ and the following source function:

$$
z(x)=\left\{\begin{array}{l}
z_{L} \text { if } x<0 \\
z_{R} \text { if } x>0
\end{array}\right.
$$

In the following we will call:

$$
\Delta z=z_{R}-z_{L}
$$

The Riemann problem is posed with the following initial conditions:

$$
u(x, 0)=u_{0}(x)=\left\{\begin{array}{l}
u_{L} \text { if } x<0 \\
u_{R} \text { if } x>0
\end{array}\right.
$$

Its solution is self similar and is made up of a standing discontinuity due to the source term jump at $x=0$ and a right travelling linear shock with speed $a$. In between the two waves there is a constant state, $u^{*}$. In view that the solution is self similar, it must be constant at $x=0^{-}$and at $x=0^{+}$. Further $u\left(0^{-}, t\right)=u_{L}$ and $u\left(0^{+}, t\right)=u^{*}$. A sketch of the solution is displayed in Figure (17). The intermediate constant state $u^{*}$ can be computed from the steady state solution of equation (101):

$$
a \frac{d u}{d x}=-u \frac{d z}{d x}
$$

After integration one obtains the exact value of $u^{*}$ in terms of $u_{L}$ and the problem parameters, $a$ and $\Delta z$ :

$$
u_{\text {exact }}^{*}=u_{L} \cdot e^{-\Delta z / a}
$$

When a numerical solution to problem (101-104) is sought by means of a time marching algorithm, the constant states to the left and right of the initial discontinuity, $u\left(0^{-}, t\right)=u_{L}$ and $u\left(0^{+}, t\right)=u_{\text {num }}^{*}$, are computed as a steady 
state solution after a few time steps, when the right running shock is away from the original discontinuity. Application of the SRNHS scheme to linear equation (101) with $a>0$ leads to:

$$
\begin{aligned}
u_{j}^{n+1}= & u_{j}^{n}-\operatorname{ar}\left(u_{j}^{n}-u_{j-1}^{n}\right) \\
& +\frac{r}{4}\left[\left(u_{j+1}^{n}+u_{j}^{n}\right)\left(z_{j+1}-z_{j}\right)-\left(u_{j}^{n}+u_{j-1}^{n}\right)\left(z_{j}-z_{j-1}\right)\right] \\
& -\frac{r}{8}\left(u_{j+1}^{n}+2 u_{j}^{n}+u_{j-1}^{n}\right)\left(z_{j+1}-z_{j-1}\right)
\end{aligned}
$$

where $r=\Delta t / \Delta x$. In order to obtain the constant state $u_{\text {num }}^{*}$ as computed by the numerical method for large $t$, equation (107) is applied to all the points in the domain, $j=1,2, \ldots, j_{L}, j_{R}, \ldots, j_{\text {shock}}$, away and to the left of the right travelling shock. Here $j_{L}$ stands for the last point where $z_{j}=z_{L}$ and $j_{R}$ for the first point where $z_{j}=z_{R}$. Note that $j_{R}=j_{L}+1$. Also $j_{\text {shock }}$ stands for the last point before the shock transition. For all points with $j \in\left[1, j_{\text {shock }}\right]$ the solution does not change with time. It can be written:

$$
u_{j}^{n+1}=u_{j}^{n}=\bar{u}_{j} \quad, \quad j=1,2, \ldots, j_{\text {shock }}
$$

Also away from points $j_{L}$ and $j_{R}$ the scheme reduces to:

$$
u_{j}^{n+1}=u_{j}^{n}-\operatorname{ar}\left(u_{j}^{n}-u_{j-1}^{n}\right)
$$

Thus for the steady state:

$$
\bar{u}_{j}=\bar{u}_{j}-\operatorname{ar}\left(\bar{u}_{j}-\bar{u}_{j-1}\right)
$$

or:

$$
\bar{u}_{j}=\bar{u}_{j-1}
$$

what leads to:

$$
\bar{u}_{j}=u_{L} \quad, \quad j=1,2, \ldots, j_{L}-1
$$

and

$$
\bar{u}_{j}=u_{j_{R}}=u_{\text {num }}^{*} \quad, \quad j=j_{R}+1, j_{R}+2, \ldots, j_{\text {shock }}
$$

since $u_{j_{R}}$ corresponds to the value of $u\left(0^{+}, t\right)=u^{*}$ computed by the numerical scheme.

For points $j_{L}$ and $j_{R}$ the scheme reads respectively:

$u_{j_{L}}^{n+1}=u_{j_{L}}^{n}-\operatorname{ar}\left(u_{j_{L}}^{n}-u_{j_{L}-1}^{n}\right)+\frac{r}{4}\left(u_{j_{R}}^{n}+u_{j_{L}}^{n}\right) \Delta z-\frac{r}{8}\left(u_{j_{L}-1}^{n}+2 u_{j_{L}}^{n}+u_{j_{R}}^{n}\right) \Delta z$ 
$u_{j_{R}}^{n+1}=u_{j_{R}}^{n}-\operatorname{ar}\left(u_{j_{R}}^{n}-u_{j_{L}}^{n}\right)-\frac{r}{4}\left(u_{j_{R}}^{n}+u_{j_{L}}^{n}\right) \Delta z-\frac{r}{8}\left(u_{j_{L}}^{n}+2 u_{j_{R}}^{n}+u_{j_{R}+1}^{n}\right) \Delta z$

where $\Delta z=z_{R}-z_{L}$ as usual. At steady state this leads to:

$$
\begin{gathered}
0=-\operatorname{ar}\left(\bar{u}_{j_{L}}-\bar{u}_{j_{L}-1}\right)+\frac{r}{4}\left(\bar{u}_{j_{R}}+\bar{u}_{j_{L}}\right) \Delta z-\frac{r}{8}\left(\bar{u}_{j_{L}-1}+2 \bar{u}_{j_{L}}+\bar{u}_{j_{R}}\right) \Delta z \\
0=-\operatorname{ar}\left(\bar{u}_{j_{R}}-\bar{u}_{j_{L}}\right)-\frac{r}{4}\left(\bar{u}_{j_{R}}+\bar{u}_{j_{L}}\right) \Delta z-\frac{r}{8}\left(\bar{u}_{j_{L}}+2 \bar{u}_{j_{R}}+\bar{u}_{j_{R}+1}\right) \Delta z
\end{gathered}
$$

And now recalling (112) and (113):

$$
\begin{gathered}
0=-\operatorname{ar}\left(\bar{u}_{j_{L}}-u_{L}\right)+\frac{r}{4}\left(\bar{u}_{j_{R}}+\bar{u}_{j_{L}}\right) \Delta z-\frac{r}{8}\left(\bar{u}_{L}+2 \bar{u}_{j_{L}}+\bar{u}_{j_{R}}\right) \Delta z \\
0=-\operatorname{ar}\left(\bar{u}_{j_{R}}-\bar{u}_{j_{L}}\right)-\frac{r}{4}\left(\bar{u}_{j_{R}}+\bar{u}_{j_{L}}\right) \Delta z-\frac{r}{8}\left(\bar{u}_{j_{L}}+3 \bar{u}_{j_{R}}\right) \Delta z
\end{gathered}
$$

which constitute a system of two equations on the two unknowns $\bar{u}_{j L}$ and $\bar{u}_{j R}$. Its solution is:

$$
u_{\text {num }}^{*}=\bar{u}_{j_{R}}=u_{L} \frac{\left(1-\frac{\Delta z}{2 a}+\frac{3 \Delta z^{2}}{64 a^{2}}\right)}{\left(1+\frac{\Delta z}{2 a}+\frac{3 \Delta z^{2}}{64 a^{2}}\right)}
$$

and

$$
\bar{u}_{j_{L}}=\bar{u}_{j_{R}} \frac{\left(1+\frac{5 \Delta z}{8 a}\right)}{\left(1-\frac{3 \Delta z}{8 a}\right)}
$$

Equations (120) and (121) above state: i) the structure of the transition at the source term discontinuity is made of one point $\left(u_{j_{L}}\right)$ and ii) the value of constant state $u_{\text {num }}^{*}$ as computed by $S R N H S$ scheme.

Note that equation (120) is the value of $u^{*}$ computed by the SRNHS scheme in terms of $u_{L}$ and other problem variables but not any discretization parameter such as $\Delta x$ or $\Delta t$. A series development of (120) for $\Delta z / a$ small enough gives:

$$
u_{\text {num }}^{*}=u_{L}\left(1-\frac{\Delta z}{a}+\frac{1}{2}\left(\frac{\Delta z}{a}\right)^{2}-\frac{13}{64}\left(\frac{\Delta z}{a}\right)^{3}+\ldots\right)
$$

which is an approximation to $u_{\text {exact }}^{*}$ (equation 106) to third order since:

$$
u_{\text {exact }}^{*}=u_{L} \cdot e^{-\Delta z / a}=u_{L}\left(1-\frac{\Delta z}{a}+\frac{1}{2}\left(\frac{\Delta z}{a}\right)^{2}-\frac{1}{6}\left(\frac{\Delta z}{a}\right)^{3}+\ldots\right)
$$


Comparing (122) with (123) it is clear that the numerically computed intermediate state is different than the true value. This contributes an error that does not diminish with mesh refining thus leading to a stagnation in the convergence rate.

The numerical solution will better approach the exact one for small values of the ratio $\Delta z /$ a that make equation (120) closer to (106), but the numerical solution will not show asymptotic convergence to the exact one with mesh refining because the scheme computes $u_{\text {num }}^{*}$ once and for all for a given $\Delta z / a$ and its value does not depend on either $\Delta x$ or $\Delta t$.

It is expected that other numerical schemes present a similar behaviour for this type of problem. For instance Bermúdez and Vázquez's numerical method [11] reduces simply to upwinding the source term when applied to this linear problem. Applying a similar analysis to the one shown above yields the result that: i) there are no internal points in the transition at the source term discontinuity for Bermúdez and Vázquez scheme and ii) the following expression for $u_{\text {num }}^{*}$ is found:

$$
u_{\text {num }}^{*}=u_{L} \frac{\left(1-\frac{\Delta z}{2 a}\right)}{\left(1+\frac{\Delta z}{2 a}\right)}=u_{L}\left(1-\frac{\Delta z}{a}+\frac{1}{2}\left(\frac{\Delta z}{a}\right)^{2}-\frac{1}{4}\left(\frac{\Delta z}{a}\right)^{3}+\ldots\right)
$$

which is analogous to (120). This means that Bermúdez and Vázquez's scheme will also lead to stagnation of the convergence rate for this problem. An example of this behaviour has been already shown in the SWE case for Bermúdez and Vázquez and McCormack TVD methods in [4].

Figure (18) shows a comparison between the exact and numerical solution to problem (101-104) for the limiting case $\Delta z / a=1$, as computed on a 102400 node mesh with SRNHS and Vázquez schemes. The numerical value of $u^{*}$ is clearly wrong for both schemes. However the rest of the solution is computed accurately thanks to the fine mesh used. Right plot represents the $L_{1}$ error convergence rate for $S R N H S$ scheme. The initial error decay corresponds to an increasingly better resolution of the solution away from the $u^{*}$ region (in particular the travelling discontinuity). It must be recalled that in the case of a linear equation a discontinuity is heavily smeared and error around it decreases fast with mesh refinement. Once the error in this region reaches the level of the mismatch between $u_{\text {exact }}^{*}$ and $u_{\text {num }}^{*}$ further reductions in it are unimportant because the global error is dominated by the latter, that remains constant, and convergence stops.

Despite this, numerical schemes based upon a true Godunov-type strategy like for instance Greenberg and LeRoux's method [31], [32] [17] made up of the following steps: 

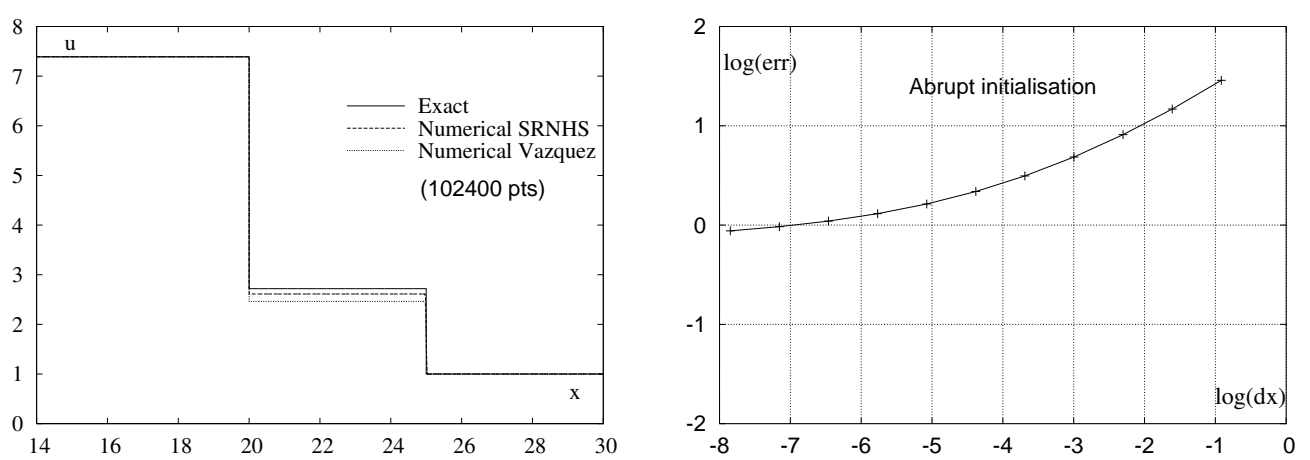

Fig. 18. Riemann problem for linear scalar equation with $\Delta z / a=1$. Initial discontinuity at $x=20$. Exact versus numerical solution with 102400 nodes (left). Error convervenge rate (right).

- Piecewise constant discretization of variables within each cell.

- Exact solution of the non-homogeneous Riemann problem at each cell interface.

- Projection (averaging) of the Riemann solution onto the cell.

should consistently approach the exact solution with mesh refining and hence uniformly converge to the exact solution. The only drawback lies in their high computational cost.

Another way of solving this problem avoiding the use of an exact Godunov method is to regularize the source term discretization (and correspondingly the initial data) to ensure that parameter $\Delta z / a$ is small at each cell interface. This can be accomplished for instance by taking:

$$
\hat{z}(x)=\frac{z_{R}+z_{L}}{2}+\frac{z_{R}-z_{L}}{2} \cdot \tanh \left(\frac{x}{C \Delta x^{p}}\right)
$$

and

$$
\hat{u}_{0}(x)=\frac{u_{R}+u_{L}}{2}+\frac{u_{R}-u_{L}}{2} \cdot \tanh \left(\frac{x}{C \Delta x^{p}}\right)
$$

instead of (102) and (104) with $C$ a numerical constant. The regularisation (125-126) introduces an error into the source function computation as well as in the initial conditions that is added to the truncation error of the scheme and the machine round off error. In the $L_{1}$ norm, the regularisation error can be easily quantified. For instance for the error introduced in the source function, $E_{z}$, one has:

$$
E_{z}=\int_{-\infty}^{+\infty}|\hat{z}(x)-z(x)| d x=C\left(z_{R}-z_{L}\right) \ln (2) \Delta x^{p}
$$

and a similar expression for the error in the initial conditions is found.

The exponent $p$ in (125) and (126) must be set slightly in excess of the asymptotic convergence order, $q$, of the numerical scheme with which the solution 

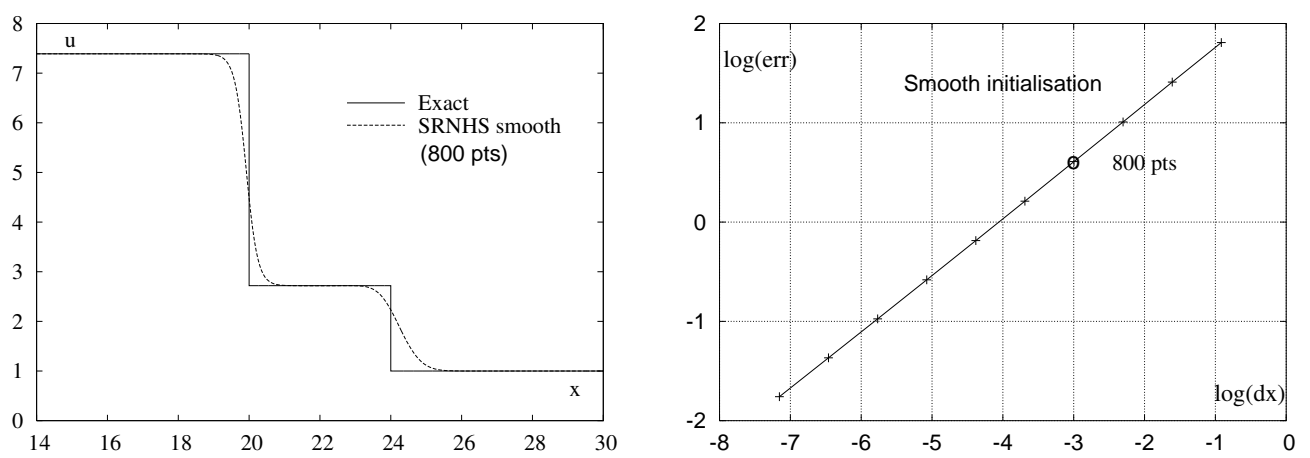

Fig. 19. Riemann problem for linear scalar equation with $\Delta z / a=1$. SRNHS scheme with smooth initialization. Initial discontinuity at $x=20$. Exact versus numerical solution with 800 nodes (left). Error convervenge rate (right).

will be computed. This is so because if $p<q$, the global error convergence will be driven by the initial and source function error due to the regularization and will mask the convergence of the scheme. On the other hand, $p>q$ can lead to faster initial convergence rates driven by the initial solution and source function regularization errors that will later, for finer meshes, fall back to the asymptotic convergence rate of the numerical scheme. This can give the false impression of a reduction of the convergence rate with mesh refinement. Put in other words: in order that the asymptotic convergence rate of the numerical method becomes apparent, the regularization of the initial conditions and the source function must be made to the same or slightly higher order of accuracy as that of the numerical scheme, $p \approx q$.

The SRNHS method is a formally first order scheme. However it is well known that first order methods applied to homogeneous linear equations with a discontinuous solution will yield only $\mathrm{O}\left(\Delta x^{0.5}\right)$ convergence [20]. Numerical tests performed on purpose with $S R N H S$ scheme on problem (101-104) with $z_{R}=z_{L}$ show exactly $\mathrm{O}\left(\Delta x^{0.5}\right)$ convergence. Hence in the linear case $q=0.5$. In order to not mask this tendency, in the following example, $p=0.6$ has been chosen.

Figure (19) shows the same test case as figure (18) computed with source function and initial condition given by expressions (125) and (126) with only 800 nodes (to be compared with 102400 nodes in Figure 18). Left plot depicts the comparison between numerical and exact solutions. It is worth noting that the $u^{*}$ state is exactly captured despite the smoothing of the transitions. Right plot shows the $L_{1}$ error convergence rate for a sequence of meshes. It is a clean straight line with 0.5 slope, as expected.

For a system of nonlinear equations the $U^{*}$ state (s) affect the configuration (speed and intensity) of the rest of the waves and hence the disagreement between the numerical and exact solutions can extend over the whole domain of integration leading to a further degradation of the convergence rate. 
Table 6

Comparison between exact and numerically computed constant states for the smooth initialisation dam break test on a 200 cell mesh.

\begin{tabular}{|c|c|c|c|c|}
\hline & \multicolumn{2}{|c|}{ Constant State 1 } & \multicolumn{2}{c|}{ Constant State 2 } \\
\hline & Exact & Numerical & Exact & Numerical \\
\hline$h$ & 3.611 & 3.602 & 2.262 & 2.260 \\
\hline$u$ & 2.102 & 2.112 & 3.355 & 3.355 \\
\hline$F r$ & 0.353 & 0.355 & 0.713 & 0.712 \\
\hline
\end{tabular}
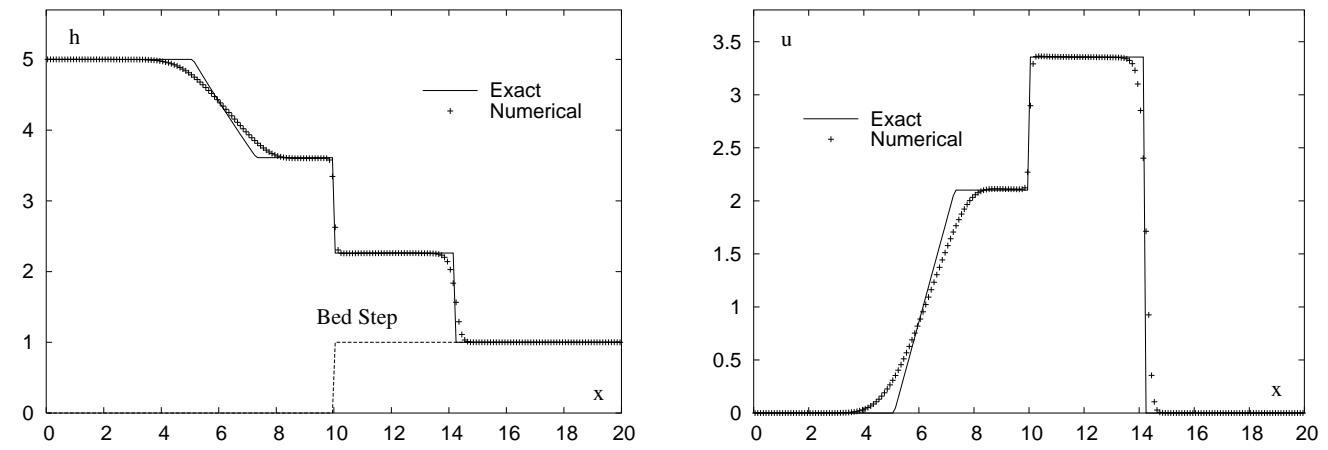

Fig. 20. Smoothed dam break problem over a smoothed step. Water depth (left) and velocity (right) at $\mathrm{t}=0.7 \mathrm{~s}$.

The same regularization procedure of (125-126) can be applied to the system case with similar results. In the following test, the dam break over a step displayed in section 6.3 has been computed with a regularized initial condition and source function. Since SRNHS scheme is a formally first order method when applied to nonlinear homogeneous problems $(q=1)$ the theoretical convergence rate, a value of $p=1.1$ has been chosen in (125-126). Figures (20) and (21) show the same problem as Figures (7) and (8) computed with the bed and initial data regularisation on the same 200 cell mesh. The solution is slightly more diffused at the expansion wave but the overall resolution is equivalent. Further the regularisation prevents the small glitches at the bed step position in the flow plot $(q)$ of Figure (8) left. The constant states 1 and 2 are correctly computed to practically the same accuracy as with the abrupt initialision run. They can be checked in Table 6 , to be compared with those in Table 2. Note however that the numerically computed states in Table 2 will not change with mesh refinement whereas those in Table 6 will uniformly approach the exact values when the mesh is refined.

Finally, Figure (22) displays the $L_{1}$ error, to be compared with that of Figure (9). The error convergence rate is a straight line with slope 1 as expected. 

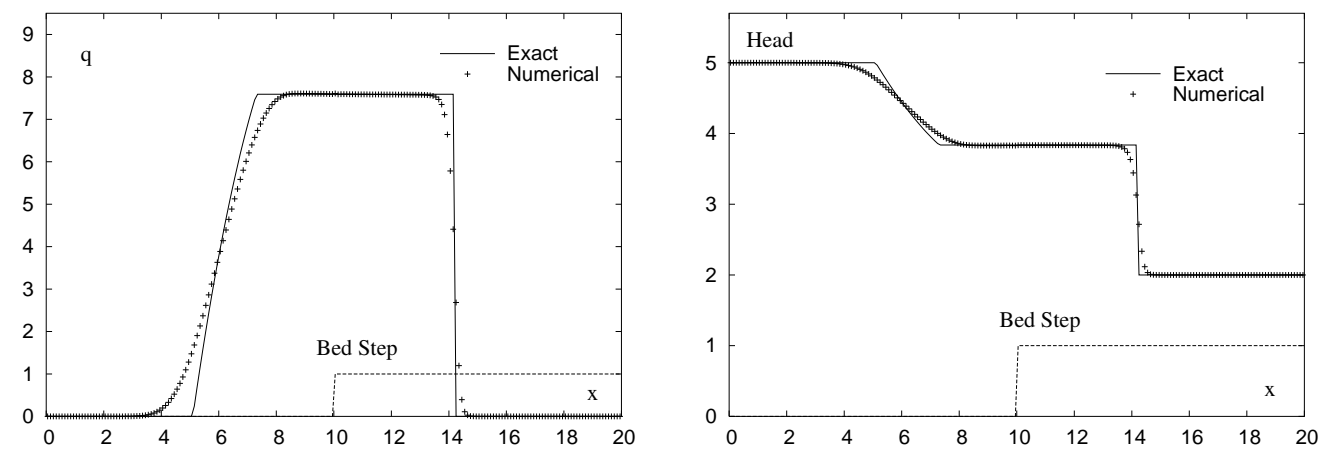

Fig. 21. Smooth dam break problem over a smoothed step. Flow rate (left) and total head (right) at $\mathrm{t}=0.7 \mathrm{~s}$.

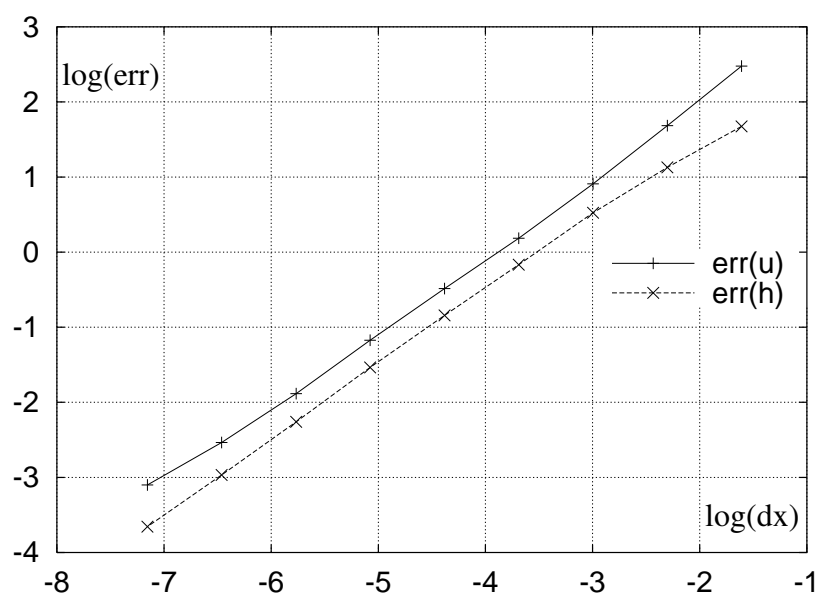

Fig. 22. $L_{1}$ Convergence plot of the velocity and the depth for the dam break over a step with smooth initialisation.

\section{Conclusions}

A numerical scheme has been presented for the computation of transient and steady solutions of systems of partial differencial equations of the hyperbolic type with source terms. The method is based upon an approximate integration of the equations over finite volume cells in two steps. This leads to the appearence of the sign matrix of the flux jacobian in the first step giving rise to a sort of upwinding of the characteristic variables. The final or updating step relies solely on physical flux and source computations instead of upon numerical flux or numerical source functions. It reverts to the so called VFRoe method in the case of homogeneous linear systems.

The scheme is computationally cheap as compared to those relying upon the calculation of an exact or approximate Riemann solution to be used á la Godunov. The SRNHS method has been analysed in the scalar and in the homogeneous linear system case and has been applied to several non linear 
problems of hyperbolic character with source terms of interest in fluid dynamics. The numerical examples presented show a good behaviour of the method for the Shallow Water and Euler equations with strong source terms forcing. In particular it has been proven that it respects the equilibrium property in still shallow water conditions. Furthermore, the construction of SRNHS method allows a natural extension to systems of partial differential equations that are not hyperbolic or that are so only within a limited domain in the phase space, such as the Modified Common Pressure Bifluid Model. The tests concerning the Ransom Faucet Problem exhibit as good resolution as other available methods for similar conditions at, in our view, less cost.

The application of $S R N H S$ scheme to problems with discontinuous source functions has led to the analysis of the so called convergence stagnation phenomenon. An explanation of this undesirable behavior as well as closed form expressions for the numerically computed constant states associated with this problem have been given for a model scalar linear equation. A regularization of the initial and source functions is proposed to cure the convergence stagnation phenomenon.

The SRNHS scheme seems to be a practical, robust numerical method for hyperbolic and quasi hyperbolic systems of partial differential equations based upon simple ideas and economy that works as well as more complex methods.

\section{Acknowledgements}

The authors would like to thank E. Audusse and A. Pascau for their critical review of parts of this manuscript.

\section{References}

[1] R. Abgrall, R. Saurel, (2003), Discrete equations for physical and numerical compressible multiphase mixtures, J. Comput. Phys., 186, pp. 361-396.

[2] Alcrudo, F., and Garcia-Navarro, P., "A high resolution Godunov-type scheme in finite volumes for the $2 \mathrm{~d}$ shallow water equation", Int. J. Numer. Methods in Fluids, 16 (1993)., 489-505.

[3] F. Alcrudo, F. Benkhaldoun, (2001), "Exact solutions to the Riemann problem of the shallow water equations with a bottom step", Computers 83 Fluids, 30, 6 , pp 643-671.

[4] F. Alcrudo, F. Benkhaldoun, (2002), "On the convergence of some numerical schemes for the solution of shallow water equations with singular source terms", 
FVCA3, HPS., R. Herbin, D.Kr oner (Eds.), pp 269-276.

[5] N. Andrianov and G. Warnecke, (2004), "On the solution to the Riemann problem for compressible duct flow", SIAM J. Appl. Math. Vol. 64, No. 3, pp. 878-901.

[6] E. Audusse, F. Bouchut, M.O. Bristeau, R. Klein and B. Perthame, A fast and stable well-balanced scheme with hydrostatic reconstruction for shallow water flows, SIAM J. Sci. Comp. 25 (2004) (6), pp. 2050-2065.

[7] F. Alouges (1998), "Matrice signe et systes hyperboliques", rapport CMLA, http://mahery.math.u-psud.fr/ $\sim$ alouges/

[8] F. Benkhaldoun, (2002), "Analysis and validation of a new finite volume scheme for nonhomogeneous systems", FVCA3, HPS ., R. Herbin, D.KrOner (Eds.), pp.269-276.

[9] F. Benkhaldoun, L. Quivy, (2006), "A Non Homogeous Riemann Solver for Shallow Water and Two Phase Flows, Flow Turbulence Combustion. 76, pp. 391-402.

[10] F. Benkhaldoun, I. Elmahi, M. Seaid, (2006), Well-balanced finite volume schemes for pollutant transport on unstructured meshes", submitted to J. Comput. Phys.

[11] A. Bermúdez and M. E. Vázquez, (1994), Upwind methods for hyperbolic conservation laws with source terms, Comp. \& Fluids 23(8), pp.1049

[12] A. Bermúdez, A. Dervieux, J. A. Desideri and M. E. Vázquez, (1994), Upwind schemes for the two dimensional shallow water equations with variable depth using unstructured meshes, Comput. Methods in Appl. Mech. Eng., 155, 49.

[13] Bon C. "Modélisation et simulations numiques d'oulements hydrauliques et de ruissellement en topographic quelconque", PhD thesis, UniversitBordeaux I, France, 1997.

[14] F. Chatelen, (1988), "Valeurs propres des matrices", M.A.M., MASSON

[15] F. Coquel, K. El Amine, E. Godlewski, B. Perthame, P. Rascle, (1997), ”A numerical method using upwind schemes for the resolution two-phase flows", J. Comp. Physics, 136, pp.272-288.

[16] Chinnayya A, LeRoux AY. "A new general Riemann solver for the shallow-water equations with friction and topography", 1999. Available from the conservation law preprint server: Available from: http://www.math.ntnu.no/conservation/.

[17], A. Chinnayya, A.Y. LeRoux and N. Seguin, (2004), "A well-balanced numerical scheme for the approximation of the shallow-water equations with topography: the resonance phenomena", Int. J. Finite Volumes, http://averoes.math.univ-paris13.fr, V 1, N1.

[18] J. Cortes, and A. Debussche, and I. Toumi, (1998), "A density perturbation method to study the eigenstructure of two-phase flow equation systems". $J$. Comput. Phy., pp 463-484. 
[19] , G. Dal Maso, P. Lefloch, and P. Murat, (1995), "Definition and weak stability of a non conservative product", J Math Pure Appl N 74, pp 483-548.

[20] , B. Depres (2003), "Lax theorem and finite volume schemes", Math. Comp. V 73, N 247, pp 1203-1234.

[21] E. Godlewski and P.-A. Raviart,(1991), "Hyperbolic systems of conservation laws", Mathématiques \& Applications (Paris), 3/4, Ellipses, pp 252.

[22] T. Gallouët and J. M. Masella, (1996), "Un schéma de Godunov approché", C. R. Acad. Sc. Paris, t.323, Série I, pp.77-84.

[23] J-M. Masella, and I. Faille, and T. Gallouët, (1999), "On an approximate Godunov scheme", International Journal of Computational Fluid Dynamics, pp 133-149.

[24] T. Gallouet, J-M Hérard and N. Seguin, (1999), Some approximate Godunov schemes to compute shallow-water equations with topography, Comput. \& Fluids, 32 pp. 479-513.

[25] , J.M. Ghidaglia and A. Kumbaro and G. Le Coq, (1996), "Une méthode "volumes finis" à flux caractéristiques pour la résolution numérique des systèmes hyperboliques de lois de conservation ", C. R. Acad. Sci. Paris Sér. I Math., V 322, N 10, pp 981-988.

[26] J. M. Ghidaglia, and A. Kumbaro, and G. Le Coq, (2001), "On the numerical solution to two fluid models via a cell centered finite volume method", European Journal of Mechanics. B. Fluids, pp 841-867.

[27] P. Glaister "Prediction of supercritical flow in open channels" Computers \& Mathematics with Applications Volume 24, Issue 7 , October 1992, Pages 69-75.

[28] , L. Gosse and A.Y. Leroux, (1996), "Un schéma-équilibré adapté aux lois de conservation scalaires non-homogènes", C.R. Acad. Sci. paris, N 323, pp 543546.

[29] , M. Grandotto , P. Obry, (1996), "Calculs des écoulements diphasiques dans les échangeurs par une méthode aux éléments finis", Revue Europénne des Eléments Finis, 5:1

[30] , M. Grandotto, P. Goldbronn, I. Toumi, P. Fillion, (1998) "Comparison of finite element and finite volume algorithms for themohydraulic studies of steam generators and nuclear reactors cores", 10th International Conference on Finite Elements in Fluids, Tucson, AZ, USA.

[31] J.M. Greenberg and A.Y. Le Roux , "A well balanced scheme for the numerical processing of source terms in hyperbolic equation", SIAM. J. Numer. Anal. 33 1 (1996), pp. 1-16.

[32] J.M. Greenberg, A.Y. LeRoux, R. Baraille, A. Noussa ir, Analysis and approximation of conservation laws with source terms, SIAM J. on Num. Anal. 34 (1997), pp. 1980-2007. 
[33] T. Gallouët, J. M. Hérard, and N. Seguin, (2003), "Some approximate Godunov schemes to compute shallow water equations with topography", Computers and Fluids, vol. 32(4), pp 479-513.

[34] T. Gallouët, and J. M. Hérard, and N. Seguin, (2004), "Numerical modeling of two-phase flows using the two-fluid two-pressure approch", Mathematical Models and Methods in Applied Sciences (M3AS), volume 14-5, pp 663-700.

[35] E.J. Hinch, (1991), "Perturbation methods", Cambridge Texts in Applied Mathematics, Cambridge University Press

[36] J.M. Hong, (2006), " An extension of Glimms method to inhomogeneous strictly hyperbolic systems of conservation laws by weaker than weak solutions of the Riemann problem", J. Differential Equations, 222, pp 515549.

[37] , M. Ishii, (1975), "Thermo-fluid dynamic theory of two-phase flows" Collection de la direction des udes et recherches d'ectricitde France, Eyrolle

[38] T. Kato, (1995), "Perturbation theory for linear operators", Classics in Mathematics, Reprint of the 1980 edition, Springer-Verlag

[39] Kurganov, Alexander and Levy, Doron, "Central-upwind schemes for the SaintVenant system", M2AN Math. Model. Numer. Anal., 36 (3) (2002), pp. 397-425.

[40] D. Kröner, M.D. Thanh, Numerical solutions to compressible flows in a nozzle with variable cross-section, SIAM J. Num. Anal., Vol.43, Is.2, pp. 796-824.

[41] A. Kumbaro, I. Toumi and V. Seignole , (2002), "Flux schemes for the numerical simulation of two phase flows", Computational fluid dynamics, Springer, pp 621-626.

[42] R.T. Lahey Jr, (1992), "The prediction phase of phase distribution and separation phenomena using two-fluid models", Boiling Heat Transfer, Elsevier Science, pp85-121.

[43] P.G. Lefloch, M.D. Thanh, The Riemann problem for fluid flows in a nozzle with discontinuous cross-section, Commun. Math. Sci. 1, (2003), pp.763-797

[44] Le Roux AY. " Discrétisation des termes sources raides dans les problèmes hyperboliques. In: Systèmes hyperboliques: Nouveaux schémas et nouvelles applications". Ecoles CEA-EDF-INRIA problèmes non linéaires appliqués, INRIA Rocquencourt (France), March 1998 [in French]. Available from: http://www-gm3.univ-mrs.fr/leroue/bublications/ay.leroux.html.

[45] R. LeVeque and H. C. Yee, "A study of numerical methods for hyperbolic conservation laws with stiff source terms", J. Comput. Phys. 86 (1990), p. 187.

[46] LeVeque, Randall J., "Balancing source terms and flux gradients in highresolution Godunov methods: the quasi-steady wave-propagation algorithm", J. Comput. Phys. 146 (1998), pp. 346-365.

[Marc 98] M. Bouker, (1998), "Modélisation numérique multidimentionnelle d'écoulements diphasiques liquide-gaz en régimes transitoire et permanant: méthodes et applications", Phd Thesis, Electricité de France 
[47] Ming-Hseng Tseng "The improved surface gradient method for flows simulation in variable bed topography channel using TVD-MacCormack scheme", International Journal for Numerical Methods in Fluids Volume 43, Issue 1 , (2003), p $71-91$.

[48] , B. Perthame and C. Simeoni, (2001), "A kinetic scheme for the Saint-Venant system with a source term", Calcolo, V 38, pp 201-231.

[49] V.H. Ransom, "Numerical Benchmark Tests", in G.F. Hewitt, J.M. Delhaye and N. Zuber, Eds, Multiphase Science and Technology, 3, Hemisphere Publishing Corporation.

[50] , P.A. Raviart, L. Sainsaulieu, (1991), " Mathematical and numerical modelling of two-phase flows", in Glowinski Editor. Computing methods in applied sciences and engeneering. Nova Science Publisher.

[51] T. C. Rebollo, A. D. Delgado and E. D. Fernandez Nieto, "A family of stable numerical solvers for the shallow water equations with source terms", Computer Methods in Applied Mechanics and Engineering, 192(2003), pp. 203-225.

[52] P.L. Roe, (1981) Approximate Riemann solvers, parameter vectors and difference dchemes, J. of Comp. Phys., Vol. 43, 1981, pp. 357-372.

[53] P. L. Roe, "Upwind differenced schemes for hyperbolic conservation laws with source terms", Proceedings of the Conference on Hyperbolic Problems (1986), p. 41.

[54] V. V. Rusanov, (1961), "The calculation of the interaction of non-stationary shock waves with barriers" JŽ. Vyčisl. Mat. i Mat. Fiz., 1, pp.267-279.

[55] S. Sahmim, (2005), " Un sch'ema aux volumes finis avec matrice signe pour les systèmes non homogènes", Ph.D. Thesis, Université Paris 13.

[56] L. Sainsaulieu, (1995), "Finite Volume approximation of two-phase fluid flows based on an approximate Roe-Type Riemann solver", J. of Comp. Phys., V 121, pp 1-28.

[57] Xing, Yulong and Shu, Chi-Wang "High order well-balanced finite volume WENO schemes and discontinuous Galerkin methods for a class of hyperbolic systems with soure terms", J. Comput. Phys. 214 (2006), pp. 567-598.

[58] E. F. Toro, (1999), "Riemann Solvers and Numerical Methods for Fluid Dynamics", Springer-Verlag, Second Edition, 624 pages.

[59] I. Toumi and A. Kumbaro, (1996), "An approximate linearized Riemann solver for a two-fluid model", J. Comput. Phys., V 124, N 2, pp 286-300.

[60] M. E. Vazquez, (1999), "Improved treatment of source terms in upwind schemes for the shallow water equations in channels with irregular geometry". $J$. Comput. Phys., 148, pp 497-526.

[61] Xing, Yulong and Shu, Chi-Wang "High order finite difference WENO schemes with the exact conservation property for the shallow water equations", $J$. Comput. Phys. 208 (2006), pp. 206-227. 
[62] J. Shi, C. Hu, C.-W. Shu, "A technique of treating negative weights in WENO schemes", J. Comput. Phys. 175 (2002), pp. 108-127.

[63] J. G. Zhou, D. M. Causon, C. G. Mingham and D. M. Ingram "The Surface Gradient Method for the Treatment of Source Terms in the Shallow-Water Equations", Journal of Computational Physics, Volume 168, Issue 1, 20 March 2001, Pages 1-25. 